NISTIR 8284

\title{
Framework and Roadmap for Smart Grid Interoperability Standards Regional Roundtables Summary Report
}

\author{
Avi Gopstein \\ Cuong Nguyen \\ Danielle Sass Byrnett \\ Kerry Worthington \\ Christopher Villarreal
}


NISTIR 8284

\title{
Framework and Roadmap for Smart Grid Interoperability Standards Regional Roundtables Summary Report
}

\author{
Avi Gopstein and Cuong Nguyen \\ Engineering Laboratory \\ Danielle Sass Byrnett and Kerry Worthington \\ National Association of Regulatory Utility Commissioners \\ Christopher Villarreal \\ Plugged In Strategies
}

This publication is available free of charge from: https://doi.org/10.6028/NIST.IR.8284

January 2020

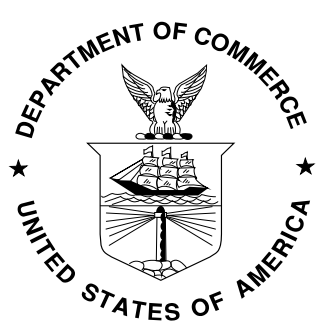

U.S. Department of Commerce Wilbur L. Ross, Jr., Secretary

National Institute of Standards and Technology Walter Copan, NIST Director and Undersecretary of Commerce for Standards and Technology 
Certain commercial entities, equipment, or materials may be identified in this document in order to describe an experimental procedure or concept adequately. Such identification is not intended to imply recommendation or endorsement by the National Institute of Standards and Technology, nor is it intended to imply that the entities, materials, or equipment are necessarily the best available for the purpose.

National Institute of Standards and Technology Interagency 8284 Natl. Inst. Stand. Technol. Interag. Rep. 8284, 58 pages (January 2020)

This publication is available free of charge from: https://doi.org/10.6028/NIST.IR.8284 


\begin{abstract}
From September to November 2018, the National Institute of Standards and Technology (NIST) partnered with the National Association of Regulatory Utility Commissioners (NARUC) to hold a series of roundtable meetings to support the revision of its Framework and Roadmap for Smart Grid Interoperability Standards (hereafter the Framework). The Framework addresses the increasingly important ability to exchange actionable information between devices and organizations.
\end{abstract}

Four one-day regional meetings were held to support development of Framework version 4.0, which reflects structural changes and increasing system complexity in the grid. Fast-paced grid evolution necessitates updated interoperability considerations, particularly as applied to the Framework’s Smart Grid Conceptual Models. These diagrams have been expanded to include four communication pathways scenarios, Legacy Utility, High-DER Architecture, Microgrid, and Advanced Bulk to be considered moving forward (shown in Appendix F). The roundtables gathered input from stakeholders (utilities, service providers, regulators, technology suppliers) on the updated Framework, as well as input on regional issues that need consideration. Meetings were organized by regions and conceptual models, as follows:

- $\quad$ The Midwest Regional Roundtable held in Indianapolis, Indiana, focused on Legacy Utility Communications. In this scenario, a logical model of legacy systems is mapped onto conceptual domains for smart grid information networks.

- $\quad$ The West Regional Roundtable held in San Francisco, California, focused on High-DER Architecture Communications. In this scenario, the generation domain (including DERs) now wraps around the transmission domain and overlaps with the customer domain, and customer devices actively contribute to system optimization.

- $\quad$ The Southeast Regional Roundtable held in Atlanta, Georgia, focused on Microgrid-driven Communications. In this scenario, the master controller becomes the key inter-domain interface. Both customer-managed and utilitymanaged microgrids are included.

- $\quad$ The Northeast Regional Roundtable held in Warwick, Rhode Island, focused on interfaces for an Advanced Bulk Grid system. This hybrid utility communications pathway scenario facilitates understanding of roles and communications of centralized, distributed, and grid edge systems.

Representatives from regional utility regulators identified key region-specific themes to provide context for further discussion.

- $\quad$ Southeast Region: Georgia is a geographically diverse state and is reliant on nuclear power, although solar photovoltaic (PV) installations are growing. This growth is completely market-driven, without any subsidies or a renewable portfolio standard, relying instead on a prudent planning process. 
- $\quad$ Midwest Region: The grid is shifting toward local generation and greater customer control over services. Utilities are shifting business models to become full-service providers. A large gap exists in consumer education to help customers better understand benefits of new technologies. Advance metering infrastructure (AMI) rollout is taking place at varying paces across the region and might be used only in billing, due to limited use cases and benefits being proposed to regulators as part of utility filings.

- Western Region: Distributed energy resources (DER) and AMI in California have seen tremendous growth, heightening concerns over interoperability, affordability, reliability, privacy, the role of utilities, and needed structural changes to the grid. Accelerating growth of electric vehicles (EV) is a major concern. As technology changes, interoperability standards will need to change as well, and places with clearly regulated assets need to have standards as part of the conversation. Regulator responsibilities are not clear in the changing and distributed grid.

- $\quad$ Northeast Region: Information technology has crept into all parts of the power sector, increasing potential to reduce costs, improve performance, and reduce risk. Interoperability is an important hedge to avoiding the costs associated with asset obsolescence. However, the quantified value of avoiding obsolescence is uncertain. There is concern about an uncertain rate of EV adoption and associated integration costs; demand management will be fundamental to keeping electricity prices stable and ensuring electrification can take place without overwhelming legacy infrastructure.

\section{Key words}

Communication, economic, distributed energy resources, interoperability standards, smart grid, and regulatory utility commission. 


\section{Table of Contents}

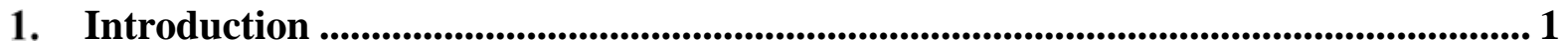

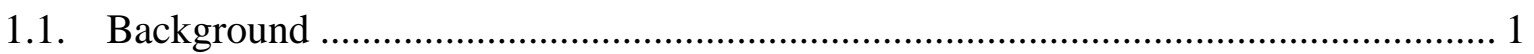

1.2. Roundtables Scope and Objectives .............................................................. 3

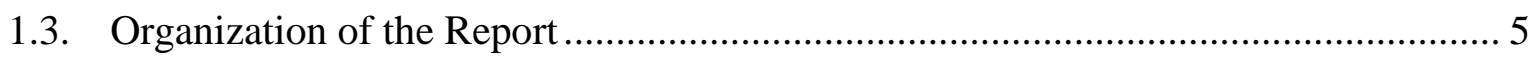

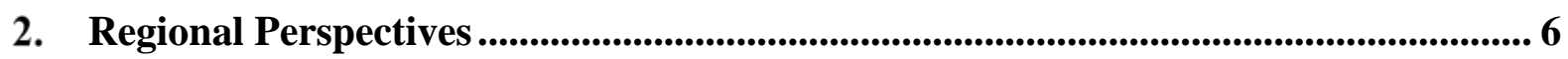

2.1. Southeast Region .......................................................................................... 7

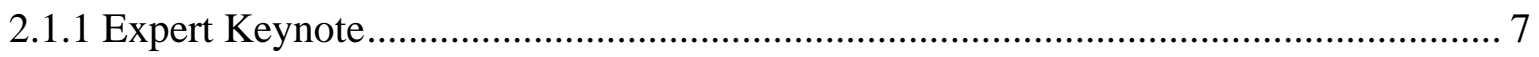

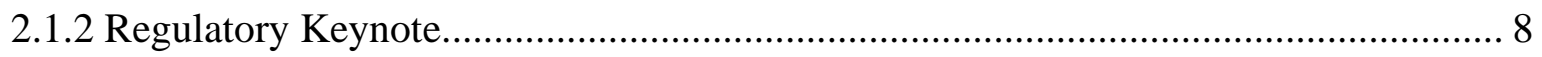

2.1.3 Panel Discussion Highlights............................................................................ 8

2.2. Midwest Region .......................................................................................... 11

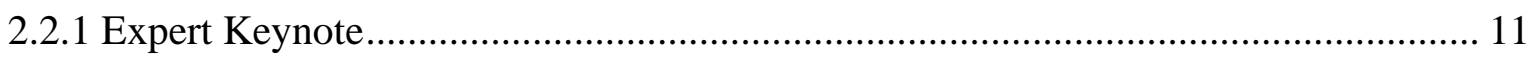

Wanda Reder, President and Chief Executive Officer, Grid-X Partners............................. 11

2.2.2 Regulatory Keynote: .......................................................................................... 12

Honorable Sarah Freeman, Commissioner, Indiana Utility Regulatory Commission ....... 12

2.2.3 Panel Discussion Highlights............................................................................. 13

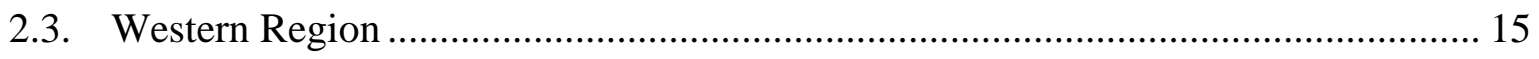

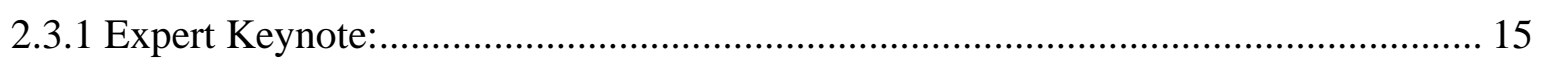

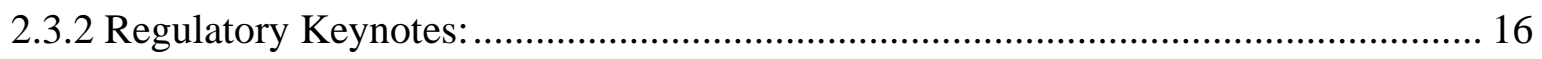

2.3.3 Panel Discussion Highlights........................................................................... 17

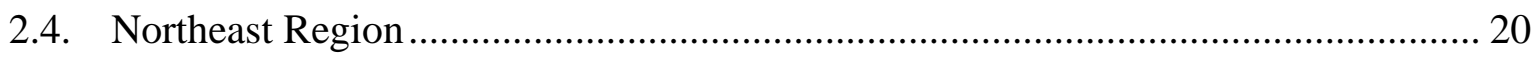

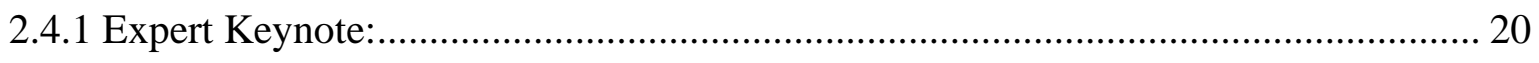

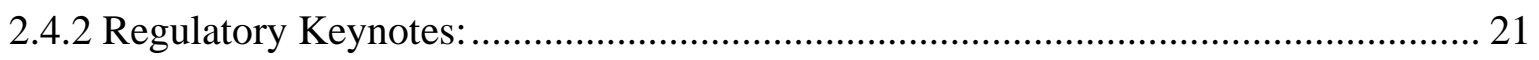

2.4.3 Panel Discussion Highlights.................................................................................. 22

3. Overarching Considerations for Smart Grid Interoperability Standards................ 25

Current and Future Issues for Interoperability Standards ............................................... 25

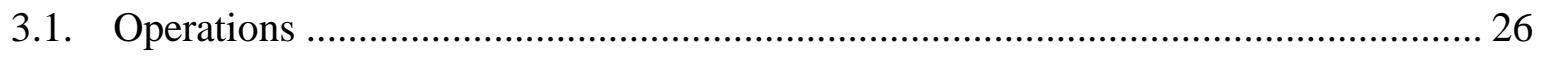

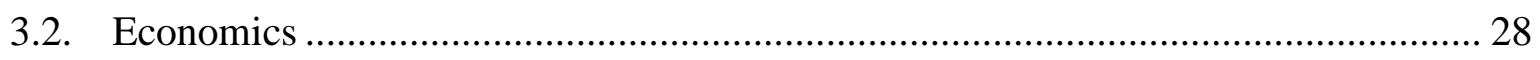

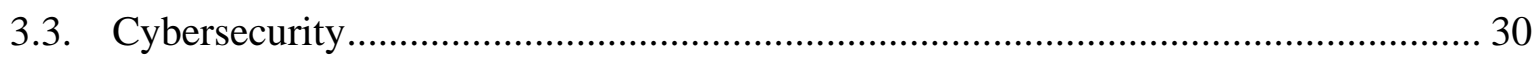

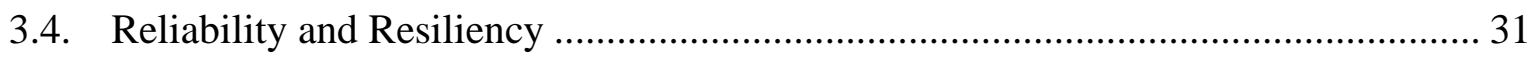

3.5. Testing and Certification ............................................................................... 31

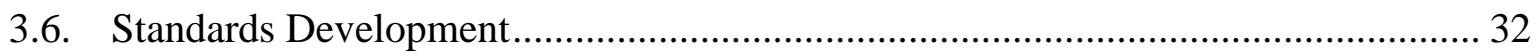

3.7. Non-Technical Challenges .................................................................................. 33

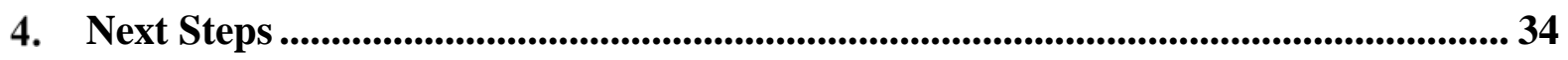




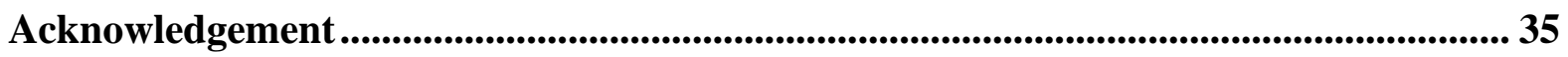

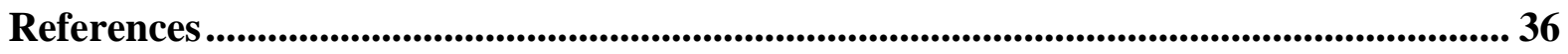

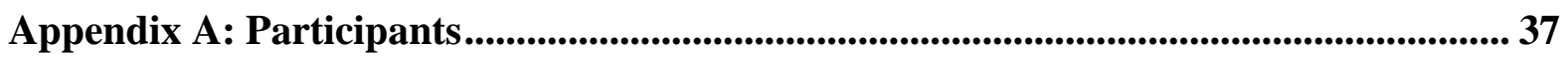

Appendix B: Regional Workshop Agendas ......................................................................... 41

Appendix C: Review Update on IEEE 1547 .............................................................. 45

Appendix D: Acronyms .......................................................................................... 47

Appendix E: Useful Links ................................................................................................ 48

Appendix F: Smart Grid Communications Scenarios....................................................... 49

List of Tables

Table 3.1 Key Overarching Issues with Impacts on Interoperability Standards .......... 25

List of Figures

Figure 1.1 Regional Workshop Locations .............................................................. 4

Figure 2.1 Proposed Schematic for Common Understanding of Interoperability......... 20 


\section{Introduction}

\subsection{Background}

As new technology modernizes decades-long practices for managing the electric grid, the ability to exchange actionable information between devices and organizations is more important than ever. From grid-edge intelligence to customer-owned resources, evolving grid architectures will introduce complex dynamics that span physical, economic, and information technology aspects of electrical systems. Improved interoperability is critical to managing these complexities. To facilitate improvements, the National Institute of Standards and Technology (NIST) in partnership with the National Association of Regulatory Utility Commissioners (NARUC) held a series of roundtable meetings to support the revision of its Framework and Roadmap for Smart Grid Interoperability Standards (hereafter the Framework).

A variety of electricity stakeholders, regions, and states are trying to address smart grid interoperability. First published in 2010, the NIST Interoperability Framework [1] was developed as the result of a specific mandate with responsibility assigned to NIST under the Energy Independence and Security Act of 2007 (EISA) [2]. The resulting interoperability framework, developed in collaboration with the broad smart grid stakeholder community, provides a way to understand, visualize, and describe the most important aspects of and requirements for interoperability and grid modernization.

Version 1.0 (Framework 1.0) provided a snapshot on the state of smart grid interoperability standards and examined the gaps in standards. It introduced the concept of grid domains to help actors and stakeholders understand where they fit within the system. Important aspects of Framework 1.0 included the separation of the power flows from the communication/information flows, and a focus on bi-directional power flows.

Version 2.0 [3], released in 2012, provided incremental updates to the first version and described progress made to address the gaps identified in the first version. The most recent version of the framework, Version 3.0 [4], was released in 2014 and introduced at a high level the increasing complexity of the smart grid as generation could connect directly to the distribution and customer domains. Prior Frameworks also included a utility communications pathways diagram to visualize and explore communications and delineate what information is exchanged between grid domains for conventional utilities. This smart grid conceptual model has been updated continually from Version 1.0 to Version 3.0.

The Framework is now being updated to Version 4.0 to reflect structural changes and increasing system complexity in the grid. The new version includes description of communications scenarios inspired by different grid architectures which are used to more closely examine unique communication and interoperability requirements, with the specific goal of exploring relationship and associated interoperability impacts expanding communications in the grid will have on four key themes: grid cybersecurity, operations, economics, and associated requirements for testing and certification. Framework Version 4.0 will reflect the variety of changes taking place in the grid, including the accelerating pace of technological change, rapidly falling prices of modern energy technologies such as solar 
photo voltaic (PV) other distributed energy resources (DER), increased proliferation of lowcost sensor and network enabled devices, and the resulting surge in granularity and amount of data being generated. The issues surrounding empowered consumers, the coordination of tens of thousands of devices operating across the system, and multi-directional power flows will be examined.

Within this context, the need to update interoperability considerations is crucial, particularly as applied to the Smart Grid Conceptual Models in the Framework. These considerations include:

- $\quad$ Explicit incorporation of DERs in the generation domain, with proximity between electric generation icons and the domains receiving the electricity.

- $\quad$ Focus on the intelligent grid, with an emphasis on increased capabilities of the distribution system. The distribution domain is now larger, more centrally located, has improved controllability, and is connected to service providers, which reflects an increasing shift from points of connection being poles and wires to servers and fault sensors.

- Increased focus on the consumer, including empowering a diversifying customerbase.

- $\quad$ Updates to the transmission, distribution, markets, operations, and service provider domains to reflect the ongoing evolution of these domains.

Framework 4.0 includes special focus on the evolving role for testing and certification as the system incorporates ever greater numbers of decentralized assets. Testing and certification is important to avoid bespoke commissioning and give confidence that the systems will perform as promised. Cybersecurity is another key consideration and was mapped to the NIST Framework with a Cybersecurity Risk Profile [5].

The Framework had a communications model diagram to complement the Conceptual Models and illustrate communications networks between domains. For Version 4.0, this diagram has been expanded to include four communication pathways scenarios to be considered moving forward (shown in Appendix F). These scenarios help to explore communication interfaces in a range of future grid systems:

1) Legacy Utility Communication Pathways Scenario: Presents a logical model of legacy systems mapped onto conceptual domains for smart grid information networks.

2) High-DER Communication Pathways Scenario: The generation domain (including DERs) now wraps around the transmission domain and overlaps with the customer domain, and customer devices actively contribute to system optimization. 
3) Microgrid Communication Pathways Scenario: Acknowledges the role of a master controller as the key inter-domain interface. Includes both customermanaged and utility-managed microgrids.

4) Hybrid Communication Pathways Scenario: This hybrid utility communications pathway scenario facilitates understanding of roles and communications of centralized, distributed, and grid edge systems.

\subsection{Roundtables Scope and Objectives}

In Fall 2018, NIST, in partnership with NARUC, held four one-day regional meetings to gather input from stakeholders on the updated Framework to ensure it reflects industry expertise and local perspectives on the evolving grid. The gathered insights and feedback help ensure that any NIST-developed guidance is reflective of the complex community of relevant stakeholders. These roundtables discussed the value and benefits of grid interoperability, impacts of interoperability on grid operations and economics, and different approaches in advancing interoperability.

The workshops were organized regionally in order to collect specific feedback, guidance, and concerns relevant to each region. The workshops were structured to include insightful speakers on the relevant topics, but also with ample time for open dialogue among the stakeholders in order to best understand the issues most important to the gathered participants. To drive discussion in these areas, each region was asked to reflect on one of the four proposed grid communication scenarios, which capture relationships between each of the domains and their components in the NIST Smart Grid Conceptual Model. The four scenarios described in Appendix F differ depending on the level and type of smart grid device deployments. 


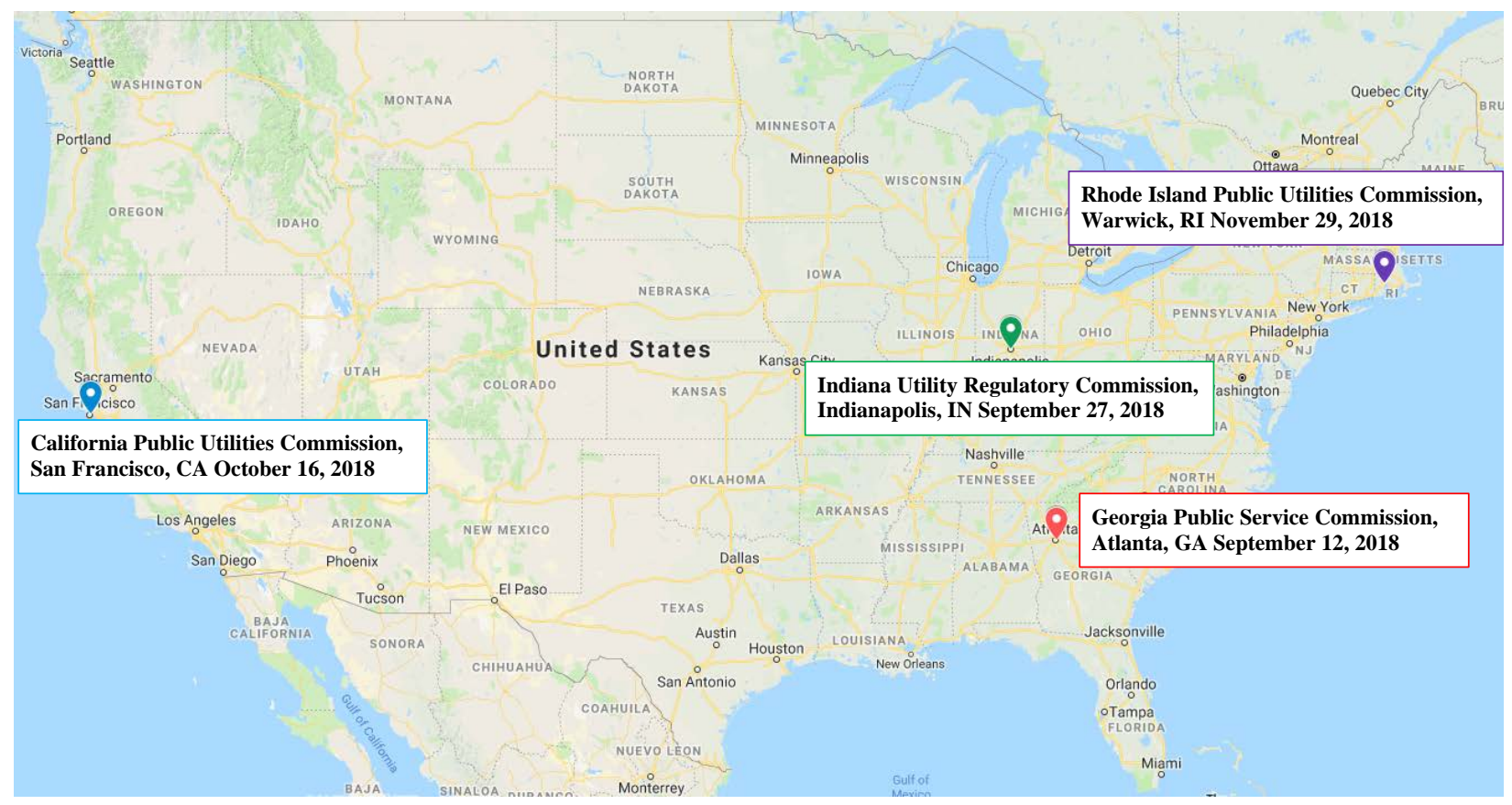

Figure 1.1 Regional Workshop Locations

The format of each workshop was similar, with keynote talks, panel discussions, and facilitated discussions on grid operations, economics and interoperability issues shaping the electric system at the regional level. Each meeting focused on examining one conceptual model relevant to each region, followed by a panel comprised of regional stakeholders. NIST staff's presentations on these scenarios are not discussed in this document but they are available on the workshop websites (see Appendix E). Facilitated discussions covered interoperability considerations and the most important interfaces for grid modernization.

- $\quad$ The Southeast Regional Roundtable was held on September 12, 2018 at the Georgia Public Service Commission in Atlanta, GA. This meeting was centered on the microgrid-focused smart grid scenario. The microgrid scenario considers two types of microgrids - one that is organized and managed by a customer or non-utility third party, and another that is organized and managed by a utility.

The Midwest Regional Roundtable was held on September 27, 2018 at the Indiana Utility Regulatory Commission in Indianapolis, IN. As the foundation for discussion, this meeting utilized the legacy utility scenario. This scenario is mapped to legacy grid systems and communications based on a conventional utility model which maintains many of the functions and characteristics of the current system.

- The West Regional Roundtable was held on October 16, 2018 at the California Public Utilities Commission in San Francisco, CA. This meeting utilized the highDER scenario as the foundation for discussion. The NIST high-penetration DER 
scenario is based on the assumption that DER deployments will accelerate into the future and will require better integration into grid operations.

- $\quad$ The Northeast Regional Roundtable was held on November 29, 2018 at the Rhode Island Public Utilities Commission in Warwick, RI. This meeting utilized a smart grid scenario focused on extending observability and control from centralized systems to the grid-edge as the foundation for this discussion. The grid scenario is based on the assumption that the impacts of expanded use of DERs will be felt throughout the electricity system-including in the Bulk Power System- and require utilities to integrate more refined observation and control at the edge of their respective systems.

Participants were given the opportunity to present their views in discussion sessions and were polled on the most relevant issues. Read-ahead materials offered an informed basis and starting point for discussion (see Appendix E for read-ahead and presentation links).

\subsection{Organization of the Report}

This report represents a factual summary of regional workshop discussions. The report includes highlights of the keynote remarks and panels (Section 2), regulatory viewpoint remarks (Section 2), as well as the key themes identified over the course of the workshops. These themes include overarching considerations for smart grid interoperability standards (Section 3) and key regional themes identified (Section 2). Next steps are outlined in Section 4. Finally, the report appendices provide: workshop participants (Appendix A), agendas for each workshop (Appendix B), summary on the review and update of the IEEE 1547 technical standard (Appendix C), acronyms (Appendix D), a list of useful links (Appendix E), and the four smart grid communications scenarios (Appendix F).

The results presented here are not all-inclusive of the smart grid community. They do represent a snapshot of perspectives based on those who attended and participated in the regionally held discussions. Attendees included a range of individuals with diverse skill sets and backgrounds (industry, academia, government, consumer advocates, and etc.) and they have expertise and interest in the topics shared at the workshop. The compiled responses from each workshop form the basis for the topical sections in this report. 


\section{Regional Perspectives}

Each regional roundtable workshop began with welcoming remarks by NIST organizers and workshop hosts, followed by an expert keynote presentation, a regulator keynote presentation, a review and update on the IEEE 1547 [6] standard, and panel discussions. The panel discussions all centered on the theme of "Perspectives on the Benefits of Smart Grid Interoperability." Each workshop then finished with facilitated discussions on key issues of relevance to the region. The following summaries provide highlights of keynotes, panel discussions, and regional specific themes.

In addition to the broadly applicable common themes identified in the discussions, each workshop also identified distinct regional issues that are equally critical to address. Each regional discussion centered on the following questions:

- $\quad$ How does interoperability relate to local operations?

- What constraints limit asset utilization?

- $\quad$ How can interoperability improve return on investment?

- What steps can be taken to maximize device and infrastructure usefulness over its physical lifetime?

Below is a list of regional themes impacting interoperability standards.

\section{Southeast Region}

- $\quad$ Optimization issues around solar

\section{Midwest Region}

- $\quad$ Customer preferences needs to be addressed at the regional level

- $\quad$ Case for AMI in rural communities

\section{Western Region}

- $\quad$ Role of DERs in Pacific Northwest

- $\quad$ Lessons learned from California testbed

\section{Northeast Region}

- $\quad$ Seasonal challenges with gas delivery

- $\quad$ Need for greater regional exchange of information 


\subsection{Southeast Region}

\subsubsection{Expert Keynote}

\section{Mark Lauby, Senior Vice President \& Chief Reliability Officer, North American Electric Reliability Corporation (NERC)}

Reliability is at the foundation of the NERC DNA. With the goal of ensuring reliability, NERC aims to determine how to clearly identify risk to the bulk power system. Key questions that NERC aims to answer include: How do we monitor and manage the changes in the bulk power system? How do we keep it reliable with so many new resources? How can new sources, especially DER, provide ramping, voltage support, and frequency response as reliably as the large centralized spinning resources have traditionally? The generation resource mix and the methods fuel delivery is undergoing a metamorphosis from large, remotely located, mostly coal, generation to newer natural gas generation and DERs.

During this transformational time in the industry, with new risks emerging due to the accelerating adoption of DERs, microgrids, and just-in-time natural gas delivery, there is a need to first identify risks to best address them. Risk identification will be aided by both experience and predictive tools - data collection, statistical analysis, and simulations of projected systems. Once understood, risks can be prioritized and mitigated through the implementation of a robust risk mitigation plan. With this focus on risk and reliability, two critical topics of importance to NERC are emerging: 1) Fuel security and 2) New types of generation.

Regarding fuel security, a diversity of fuels strengthens resilience and allows for stronger responses to shocks (e.g., Fukushima nuclear disaster, Aliso Canyon gas leak, rail strikes, etc.). As the industry transitions to new types of generation, reserve margins for generating capacity are becoming less important, but energy availability for these generation sources becomes critically important to managing risk. For example, rather than simply building new generation to reduce outage risk, natural gas fired plants must be assured of their natural gas supply. Furthermore, the benefit of additional capacity is dependent on the availability of the gas supply. Different sources like wind and solar can also contribute in new ways to frequency response, but the protocols and capability must be included in the interconnection agreements. With this growth in new types of technologies, we still need to do a better job of understanding the interdependencies between markets and regions - for instance, disruptions in Texas gas markets have an impact on operations and economics in New England. Digital controls can create new operational and economic benefits, but also can introduce risk due to cybersecurity issues or the potential for unanticipated response to control signals. As the grid transitions to more distributed generation technologies, it is critically important that the devices that manage this new generation mix are interoperable with existing and future systems. 


\subsubsection{Regulatory Keynote}

\section{Honorable Lauren “Bubba” McDonald, Chairman, Georgia Public Service Commission}

Georgia is a geographically diverse state, with a wide variety of landscapes for the utilities to operate including coastal, mountain, and agricultural areas. Georgia also is reliant on nuclear power, with two reactors and two additional reactors scheduled for completion in the next few years. Affordable and reliable power is particularly essential for businesses. Solar PV has grown rapidly in the past 5 years from nearly zero installed solar to more than $1.5 \mathrm{GW}$ today. The Georgia PSC Commissioners are elected by Georgia voters. The growth in solar is market driven, without any subsidies or a renewable portfolio standard, relying instead on a prudent planning process with Georgia utilities. The majority of the state's solar PV is utility scale, with some rooftop solar as well.

\subsubsection{Panel Discussion Highlights}

\section{Jamie Barber, Georgia Public Service Commission}

Regulations are developed with the goal of providing consumers with safe and reliable power. Accordingly, the key interoperability considerations for the Commission are limited to two questions: "What is going to provide safe and reliable service?" and "What is going to be reasonably priced?” Cost is key, but regulators also require quantification of the benefits. It is also important to consider features and services, not specific technologies. Getting all stakeholders involved will help to ensure these questions are being answered with the best information.

\section{Bill Colavecchio, Underwriters Laboratories}

Interoperability has always existed in some form-for example Wi-Fi or electrical outlets and receptacles which each allow interchangeable products from different vendors to run on them. It is important to realize that we do not have to get to a single standard - a finite number of solutions is acceptable. Instead, the critical interfaces and situations where standardized interoperability delivers significant benefit need to be identified. The collective vision for utilities is widespread interoperability based on a limited number of standards, which give vendors flexibility. Utilities need to have flexibility as well; if a utility is locked into a specific vendor, they have no cost leverage. Thus, without interoperability, utilities will not be able to use newer and more innovative technologies from other vendors. Additionally, even when devices are compliant to the standard, devices may not communicate with each other because they comply with different parts of the standard.

\section{John McDonald, General Electric Grid Solutions}

The importance of interoperability is especially clear when devices and components from different vendors are integrated into a single solution. The individual components need to be able to communicate with each other for the devices to function. This importance is also reflected in the cost of a typical distribution management system (DMS), for which 70\% of 
the cost is for proper integration with other parts of the operations center on the back endonly $30 \%$ of the cost is in the DMS itself.

The Smart Grid Interoperability Panel (SGIP) was formed in 2009 to address the interoperability problem with 25 members from 22 different stakeholders. A challenge that SGIP attempted to address is that the development of new standards should not take over 5 years' time. The SGIP sought to develop a process that would fast-track standards development so that development could be done in less than 2 years. Within SGIP, the Priority Action Plan (PAP) process worked well to help to quickly and creatively identify new solutions to interoperability problems. Compliance to standards does not imply interoperability; for this reason, "Plugfests" at regular intervals are also needed to ensure interoperability. In the past, SGIP would examine all the standard organizations' activities to help coordinate them. With technology change accelerating, the coordination role is very important going forward. Independent testing is also needed to ensure interoperability.

\section{Howard Smith, Southern Company}

The grid is rapidly evolving, moving from analog systems with equipment designed to last 40-60 years to digital systems with equipment that is replaced much more frequently. As utilities select technologies, they need to make smart and informed decisions that take into consideration several factors, including the following:

- Costs: What is the cost of new protocols and what will the impact be on customer rates? Cost must be factored into the decision making - it cannot be done purely on technical merits.

- $\quad$ Obsolescence: With technology rapidly evolving, how long will an investment in a technology be relevant before it needs to be replaced by another technology? For example, utilities that invested early in advanced metering reading (AMR) may need to invest again in AMI.

- Cybersecurity: If a new technology is rolled out that results in customer information getting lost, the utility will lose customer trust and support for similar technology upgrades in the future. It is thus critical to address this issue and embrace robust cybersecurity in the protocols now.

- Data privacy: Similar to cybersecurity, this is critical to maintain customer trust and needs to be taken into account during protocol development.

- Reliability: If a traditional system is not being built, the new system needs to have the same level of assurance that it will perform to the level of the old system.

\section{Summary of Southeast Region Panel Q\&A}

The question and answer session included discussion of the following points:

- $\quad$ Regional variance of interoperability requirements: Regional variations of standards that address similar requirements can create additional costs. For example, in California, the public utility commission (PUC) required that customers be able to move with their device across territories (e.g., from San 
Diego to Northern California). For some GE smart appliances, the same devices were sold across the United States, but they needed modules that could be added (e.g., HomePlug, Wi-Fi, ZigBee) to make it work in the state it was sold or if states changed which communications protocols were supported. The heterogeneity made the device more expensive. Electrical meters in South America also face regional variation, where some countries use International Electrotechnical Commission (IEC), some use IEEE, and Brazil relies on neither exclusively.

- Importance of $R \& D$ as a test bed for interoperability: One $R \& D$ project in Birmingham Alabama uses a 52-home smart neighborhood connected to a microgrid and community solar. This neighborhood allows researchers to test controllers and collect data to test and optimize how the equipment and systems work together. Importantly, the utility is working directly with the developer and the results will be shared with industry, with developers adopting the best results into the design practices and features of their future communities. It is a long-term change. Georgia Power is looking to run a similar R\&D project in its service territory with condominiums next.

- $\quad$ Motivation for interoperability: For vendors, the best motivator for interoperability standards is the utility. These standards should be market driven, not mandated by the government. Customers need to require any additional functionality. One challenge is that it often requires customer education to make sure customers understand the benefits of the additional functionality, which can be costly and challenging.

- Interoperability costs: With rising costs, it is important to quantify concretely the benefits of interoperability, otherwise interoperability is always at risk of being cut because benefits are not well-captured. In some cases, the benefits can help justify a project - like improved reliability, enhanced capabilities, or enabling flexibility in technology adoption. For example, consider a utility that is buying a new supervisory control and data acquisition (SCADA) system, DMS, or energy management system (EMS). While the database that feeds the SCADA system can be proprietary, requiring a common information model (CIM) [7] interface opens a realm of possible applications. Another related issue is that digital technologies are generally experiencing a shorter life cycle, and thus the costbenefit analysis is more difficult to make compared to long-lasting analog devices of years past. In the analysis, the utility may need to assume multiple generations of digital devices to match the lifetime of the analog devices and allow for a fair apples-to-apples comparison. Policies and guidance for performing cost-benefit analysis may vary from state to state, affecting which factors are included in the decision making for cumulative net present value requirements. 
Broader themes emphasized in the Southeast workshop discussions included the following:

- Florida, despite being the "Sunshine State," sees much greater cloud coverage than other sunny states like Nevada, reducing the appeal of solar PV.

- Hurricanes create potential for widespread damage and outages, which could uniquely affect the ability to bring DER back onto the grid following a storm.

- Utilities have tended to implement technologies on their own without a significant concern about the role of third parties, and handle interoperability internal to the utility.

- Utilities are providing solutions directly to customers and installing technology on behalf of customers to pilot new technologies.

\subsection{Midwest Region}

\subsubsection{Expert Keynote}

\section{Wanda Reder, President and Chief Executive Officer, Grid-X Partners}

Interoperability is the key to unleashing more value in the electric grid. Ms. Reder emphasized several drivers and trends as becoming increasingly important to the industry at large:

- Changing customers. Customers are more dependent on electronics and care increasingly about choices and information.

- $\quad$ Aging infrastructure. How to evolve infrastructure utilization rather than replace it as technology evolves

- $\quad$ Aging workforce. As the baby boomers retire, how will the industry transition to a younger workforce

- $\quad$ Environmental requirements. Using energy in a sustainable way.

- $\quad$ Security. Greater risk as more equipment and processes are deployed.

- $\quad$ Distributed energy resources. With the supply mix changing through accelerating DER deployment, it is especially important to determine how to best prepare.

Ms. Reder presented a high-level framework for examining and understanding the changing grid, in terms of Making, Moving, and Using energy. Making energy (i.e., energy generation) is becoming more distributed. Moving energy (i.e., transmission and distribution) is becoming more and more essential, with the increasing decentralization of energy generation. Using energy (i.e., energy end-use) is changing such that power producers now need to consider what customers are doing behind the meter (e.g., demand side management, solar PV, shifting loads)

With increasing DER penetration, the grid is evolving from business-as-usual operation with one-way power flow, to a push for utilities to develop suitable infrastructure to enable adoption and installation of new DER, to a future state dominated by local generation and two-way power flow. This future state is reliant on innovation in resource flexibility, power electronics, distributed intelligence, and adaptive protection. With this change requires an 
understanding that the way electricity is generated is different than traditional means of generation, and that the distribution utility will have to better understand what is going on behind the meter. In the Midwest, however, PV penetration has lagged other regions despite falling costs of installations. That lag represents an opportunity to gain insight from the experience of other regions and how to incorporate DER reliably while adding value.

Two examples were presented to illustrate the critical role of interoperability in providing value stacking in the grid. In the first example, Ms. Reder described the situation in Minster, Ohio, which gets its wholesale power from PJM. In response to a frequency response initiative from PJM, Minster hired a 3rd party to set up a facility to run the frequency market and provide power to the Minster community [8]. This program reached financial break-even in years $3-4$, without any outside incentives. Interoperability is a key enabler to making this program work well because the frequency market requires sub-cycle response times, so market participants have an incentive to maintain fast communication and response capabilities. The second example focused on water heaters. If smart switches can coordinate among all the different water heaters in a neighborhood, value can be derived. If the water heaters have poor interoperability, the value to the grid is greatly diminished.

\subsubsection{Regulatory Keynote:}

\section{Honorable Sarah Freeman, Commissioner, Indiana Utility Regulatory Commission}

Developing a fully interoperable smart grid that best serves the public interest requires the involvement of a broad range of stakeholders. A major grid trend is reflected in a shift towards local generation and greater customer control over services. In Indiana, the uncertainty associated with the accelerating growth of electric vehicles is a major concern. The Commission is working closely with utilities and the Indiana Energy Association to best determine what increased EV growth will mean in Indiana, which is a car-intensive state. It is not clear at this time how EVs will shift peak and if EVs will require advanced rate design or meaningful time-of-use rates. It is essential for state and federal regulators to coordinate their efforts on this topic, or risk stifling innovation.

Human behavior is an important aspect broadly impacting the grid space, in its many levels of sophistication and technologies. It is expected that customers will use technology in unpredictable ways that may or may not match their designed use. It is imperative that regulators become nimbler to address the rapidly changing technology landscape.

With respect to the roll-out of AMI, this is taking place at its own pace across Indiana. In some cases, AMI may only be used for improving billing, which makes it difficult to build a compelling cost-benefit case. (No utilities in Indiana took stimulus funding in 2009.) There is also a fair amount of customer resistance to AMI types of products, chiefly due to privacy concerns. Choice is essential for customers. Utilities are shifting their business models from the traditional linear model to becoming full-service providers, with many utilities setting up online marketplaces to offer products to make customers' homes and workplaces smarter. More needs to be done though, as there is a large gap in consumer education to help them better understand the benefits of the new technologies. 
Customer preferences needs to be addressed at the regional level: Customers in the Midwest tend to be risk averse, practical, frugal, engaged, and generally trusting of the utilities.

Because of the tendency to be risk averse, there needs to be a focus on customer education and communication to better understand the benefits of interoperability. There needs to be broad consensus before early adopters will be willing to adopt new technology. This situation can be different in other states.

\subsubsection{Panel Discussion Highlights}

\section{Russ Desalvo, Commonwealth Edison (ComEd)}

Smart devices are an important entryway into the smart grid for utilities. For example, with ComEd, smart grid investments began with the Illinois Energy Infrastructure Modernization Act of 2011. This investment was used to install smart meters across the state, which allowed not only utility operations to benefit from the new meter data, but also customers to see their usage data. ComEd's next step is to integrate AMI water meters and smart street lights. The Illinois Future Energy Jobs Act of 2016 was also an important stimulant, helping accelerate solar adoption in the form of community solar (up to $2 \mathrm{MW}$ each). Residential solar applications tend to cluster around specific areas and specific feeders. ComEd's Bronzeville microgrid project is also piloting a variety of smart grid initiatives, including EV charging, demand response, and building management systems. This project is owned by the utility, but its generation resources are owned by a third party.

\section{Ed Eckert, Itron}

The choice of communications infrastructure is a strategic decision. To help with understanding interoperability, the following analogy may be useful:

Standards can be remarkably flexible and therefore do not guarantee interoperability. In this sense, standards are like grocery lists where the same list of ingredients could be used in wholly incompatible recipes. Recipes provide more specific direction and, in this analogy, are like interoperability profiles, which provide guidance for implementing the standard on a device to ensure interoperability. Finally, cookbooks are like architectures or information models, which help to create a family of interoperable devices.

\section{Lynne Kiesling, Purdue University}

In the past, the grid comprised only a few layers of services which may have been vertically integrated to control the transaction costs between them. The complexity of the modern grid is adding many more layers that then must interact. Interoperability enables more seamless exchange between layers for mutual benefit. Digital platforms enable the monetization of excess capacity in the grid by reducing transaction costs. The internet is a good example of how a technology can reduce transaction costs, and companies like Uber or Airbnb are a good example of a digital platform business model. Similarly, interoperability reduces 
transaction costs and enables parties to make resources available for exchange, which has implications for business models in the industry. It can open up new markets and can lead to further unbundling of the vertically integrated supply chain.

\section{Kenya Stump, Kentucky Office of Energy Policy}

The Kentucky Office of Energy Policy is a non-regulatory entity that provides policy support to the state commission. In assessing the benefits of interoperability, there are several fundamental questions that need to be answered:

- $\quad$ Is it useful for customers and prudent?

- How does it impact reliability of the service?

- $\quad$ Is it at a fair and reasonable cost?

With a cost-based regulatory structure, a monetary cost/benefit number must be demonstrated, which often makes it difficult to incorporate qualitative benefits like interoperability. Prior to including them in the rate case and getting approval from the commission, it is essential to unlock any value streams and show how they will benefit the customer. As a result, benefits like cross-functionality and improved internal efficiencies need to be translated into the language of the regulator. What are the customer benefits of improved internal efficiencies or cross-functionality? If the benefits do not accrue to all customers, then the commission will have to scrutinize it very carefully. In some cases, utilities have programs that are cost effective, but it is not clear how customers benefit.

\section{Summary of Midwest Region Panel Q\&A}

The question and answer session included discussion of the following points:

- $\quad$ Communicating interoperability benefits to a regulator: Need to unlock additional value streams and convey additional layers of interoperability value to the regulator. For example, light emitting diode (LED) street lights have a clear customer cost-saving value; in addition there is a small incremental cost to include technologies (including those that support interoperability) that enable additional value to be derived from smart city functionality.

- $\quad$ Interoperability metrics: Current metrics for interoperability are largely inadequate. Need to think carefully about how to define and quantify appropriate metrics. For example, for increased internal efficiencies, benefits are more than just fewer people going to read meters. What are the internal learnings? Need to also give time to demonstrate benefits that may not accrue immediately. The challenge is that while it might be difficult to have enough available information showing positive benefits to customers in order to get regulatory approval, evidence suggests that unanticipated benefits ultimately will accrue to customers. Capturing previously uncaptured value streams is very difficult in a least-costbased regulatory environment. Utilities will need to estimate counterfactuals and compare what would happen without interoperable devices versus what might 
happen with them. That comparison relies on models and estimates, which are inherently subject to critique. For example, improved load forecasting has been identified as a key qualitative benefit of AMI—but it needs to have metrics associated with it.

- Value that interoperability brings to the grid: Interoperability brings great value to the following devices and systems: 1) Customer systems, 2) Interface of generation (DERs) and the distribution system, 3) Communications architecture like distribution interfaces, and 4) Human interfaces.

Broader themes emphasized in the Midwest workshop discussions included the following:

- Case for AMI in rural communities: For many of the rural communities in the Midwest, low population densities mean a high average cost of (traditional) metering. AMI has the potential to decrease system-wide costs, but the business case must be made for AMI in utility filings with the commissions.

- Utility opposition to consumer-delivered electricity: Investor-owned utilities in some midwestern states have opposed the delivery of consumer-generated electricity into the grid.

\subsection{Western Region}

\subsubsection{Expert Keynote:}

\section{Don Von Dollen, Senior Program Manager, Electric Power Research Institute (EPRI)}

The focus of Mr. Von Dollen's talk was on the definition and practical work that goes into achieving interoperability. This work is taking place in the context of the following overarching smart grid drivers and trends: 1 ) Shifting customer expectations (e.g., receiving quicker updates on any outages), 2) Cleaner generation with greater integration of renewable energy and coordinating devices to help optimize the grid, 3) Greater resilience to enable quick recovery from natural disasters and cybersecurity threats, 4) Aging infrastructure and workforce with 40-60 year traditional utility infrastructure in need of refreshing, and 5) Smarter Cities that provides great new functionality (e.g., real-time monitoring, smart traffic lights, etc.) but also introduces new third parties to the grid marketplace.

The most basic definition of interoperability is the ability for two devices to exchange useful information and work together. In practice though, there are many degrees of interoperability, with interim stages that lead to plug-and-play interoperability. The farther the distance between interoperability, the greater the costs to integrate disparate technologies. Strong communications and stakeholder engagement are key to achieving interoperability, with the variety of relevant stakeholders including standards development organizations (SDOs), government agencies, alliances, user groups, and private R\&D organizations. As new players emerge and operations become more complex, interoperability takes on additional importance. 
Interoperability ultimately relies on the development, refinement, and adoption of a standard. Development is a time-consuming process that involves all the key stakeholders, each with their own particular motivations and preferences. SDOs are often assisted by government agencies (e.g., national laboratories, NIST, NYSERDA, CEC, etc.) to do the technology development. Following the development of a standard, the process continues through the refinement of the published standard with the assistance of alliances and user groups. These groups develop a profile around the published standard and develop reference implementations of the standard. Refinement also includes interoperability testing, such as the IEC 61850 interoperability plugfest, which is hosted by alliances or user groups. Vendors test the system against their prototype software, aided by witnesses such as consultants and potential customers, with the aim of exchanging information with the developed profile. The learnings from these tests go into the refined standard, which then undergoes third party certification testing to validate that the product conforms to the profile.

The last stage before interoperability can be achieved is the large-scale adoption of the standard. However, unless a customer asks for a particular standard, suppliers may not include it in their product. Therefore, to aid in standard adoption, utilities and regulators have a role to play in aiding the adoption of a standard, by encouraging suppliers to include the standards in their products. Ultimately, interoperability will save time and money for utilities, however it is a long and difficult process to get to true plug and play interoperability.

\subsubsection{Regulatory Keynotes:}

\section{Honorable Carla Peterman, Commissioner, California Public Utilities Commission}

There has been tremendous growth of DERs in the utility service territories regulated by the California PUC, including over 6,500 MW of distributed solar on rooftops (more than the level of nuclear energy serving the state of California) and accelerating electric vehicle penetration. This has heightened the need to think about how to best implement interoperability, as well as ensure the affordability and reliability of these distributed resources. The state is also seeing great growth in its smart metering infrastructure, which raises important questions on privacy concerns, the role of utilities, and what customer information needs to be collected. The state is examining what structural changes may need to occur to the grid and is analyzing how to best integrate these resources into the grid. A common understanding is needed.

The development of interoperability standards is needed to best coordinate, guide, and manage the changing electric grid as it copes with increasing changes in technology, economics, and customer behavior. As technology changes, standards will need to change as well, and places with clearly regulated assets need to have standards as part of the regulatory approval process. The regulatory process will rely on developing multiple future scenarios of the grid. Through channels such as regulatory proceedings, utilities can direct the discourse around the changing grid, anticipate oncoming issues, respond to issues, or nudge the market. Even if the development of standards is anchored on strong technical principles, if the standard is seen as the catalyst for a change to the grid, it can become a contentious issue that runs the risk of failing. With this new market emerging, regulators may potentially be taking 
on the demands of a whole new market, acting as a regulator and dealing with disputes. However, the regulator can only be responsible for something it controls, which is something that is not necessarily clear in the changing and distributed grid. For example, currently IOUs determine what grid investments to make, which may change. The edge of the regulatory map is also getting blurred as third parties gain access to the grid and provide services to utilities or other customers (i.e., a "prosumer" selling solar electricity kWh to a neighbor).

\section{Honorable Jeffrey Ackermann, Chairman, Colorado Public Utilities Commission}

Another responsibility of public utility commissions is to control the pace of change to account for policy attitudes. The Colorado PUC is expected to issue a rulemaking that will address a variety of topics including interconnection, solar, storage, and qualifying facilities. In Colorado, NERC also is a key stakeholder on the topics of standards and reliability. NERC has standards it oversees and has the power of enforcement and fines that can be stronger than the state. Thus, the state serves as a backstop and complementary role to NERC and does not try to write NERC-like standards.

\subsubsection{Panel Discussion Highlights}

\section{Adam Danise, Public Utilities Commission of Nevada}

Nevada lags behind California with respect to the development of DERs. Prior to 2017, Nevada only reviewed distribution costs when it came through a rate case. A utility was then required to file a distributed energy plan with the commission. On the distribution side, feedback is needed from the industry on how to best evaluate the benefits of DERs. On the transmission side, a Nevada utility led an RFP for dispatchable renewable energy; however, feedback is needed on how to best leverage interoperability there.

\section{Roy Lum, Pacific Gas and Electric (PG\&E)}

PG\&E is investing heavily into its grid modernization program and research design and development (RD\&D), to aid in the replacement of existing systems with DERs. Legacy SCADA systems are being replaced, while advanced distribution management systems are being integrated to better visualize and control DERs. Standards will play a very important role in enabling DERs and preparing for the future. PG\&E is looking to gain practical experience and learn from other utilities.

\section{Devin Hampton, UtilityAPI}

UtilityAPI was founded by people doing solar installations who found that standards and the data being produced were not robust enough to put into models. A standardized way to collect, interpret, and analyze data across the country was clearly needed, not only for solar, but also other grid-connected systems. The challenge is that often DERs and utilities want each other's data, but don't want to share their own data. To solve this challenge, it is important to show that sharing data is safe and secure and that every part understands what they are doing. 


\section{Anne Smart, ChargePoint}

In California, there is clear EV charging policy with respect to standards, whereas some other states are not as advanced in their EV policy making (e.g., some jurisdictions still collecting data on whether regulating charging stations are within their jurisdiction). ChargePoint has over 55,000 charging points in the U.S., providing hardware and network services, but do not have ownership over the stations. Interoperability for EV charging is important, especially in jurisdictions where EVs and/or EV charging stations are included in the definition of DERs. For example, EVs should not just be considered storage and limited by the standards and requirements that are developed for storage. Again, emphasizing the need for policy to be characteristic-based and not technology-based. For drivers, while grid services may be of interest, their key concern is that their car gets charged regardless of any demand response events. Interoperability ensures the vehicle can connect for electronic refueling.

For a company like ChargePoint, multiple interest groups want to interact with them, including utilities, car manufacturers, and banking industries while also ensuring a positive customer experience. Interoperability for EV charging is relevant at 4 levels:

- $\quad$ Car to charging station/point/port - How is car communicating with the station and ensuring a charge?

- $\quad$ Hardware to software - How sensors and technology can analyze physical properties and make decisions, along with the grid.

- $\quad$ Network to network - How to enable a driver to use ChargePoint (the hardware and network services) at different ports?

- $\quad$ Network to grid - How does car communicate with the grid?

Currently, the California Energy Commission has proposed some standards for receiving grants. Moving forward, ChargePoint wants interoperability to protect a fully competitive marketplace - currently not all EV charging companies may be aware of the standards activities involved. Also, ChargePoint believes that embedded meters should be in all stations; testing protocols are needed; and standards must be applicable to new technologies (i.e., charging companies do not want to force a new technology into an old standard).

\section{Lorenzo Kristov, Independent Consultant}

For interoperability on the grid, there is the potential for many interfaces that may benefit from (multiple) standards. There would be a benefit to prioritization, but the challenge is to accomplish this with a multi-layered, multi-phased system. How to prioritize something where there are numerous connection points? This question is key for distribution utilities as we move into a high-DER future. The key intervention points need to be determined where setting standards opens up new possibilities and functionality. Then economies of scale would enable more replicability.

The notion that the Independent System Operator (ISO) is the central optimizer of everything is business-as-usual thinking. Rather, if there was a new business manager that managed at the DER level, it could be a Distribution System Operator (DSO) that sees all the operations 
and wires and determines how to optimize resources to give the ISO what it wants and manage the market.

Resilience still needs to be examined at a utility scale. However, developing standards for community-level resilient supply systems could be a next best step, particularly if it could be replicated across the country. This thinking ties into preparation for climate events as wellstandards may be able to help accelerate resilient projects and make it easier to scale and/or replicate the solutions.

\section{Summary of Western Region Panel Q\&A}

The question and answer session included discussion on the following points:

- $\quad$ Roles and responsibilities for grid modernization: In the context of grid modernization investments for DERs, PG\&E provides the network and telemetry (communications) to enable DERs and is making investments to ensure future functionalities for the DERs. Data and customer integrators like UtilityAPI are trying to create an infrastructure that enables stakeholders to have access to who sees what, when, and where. For example, customers could give permission to the utility or DER provider to view data.

- Data availability and sharing issues: The need to share data depends on what is trying to be accomplished - relevant parties need to identify what information is needed; set design targets; and develop collaborative processes to implement the design ideas.

Broader themes emphasized in the Western workshop discussions included the following:

- Role of DERs in Pacific Northwest: The hydro resources of the Pacific Northwest enable cheap and reliable power, yet many customers still want DERs. Planners need to take into account the perspective of what generates value for customers, along with how to best align with what utilities need.

- Lessons learned from California testbed: Lots of lessons learned from AMI investments that can be used and leveraged by other states for things like helping to determine critical system requirements. There is interoperability across the power system that allows the use of grid data to get near-real time data for demands and needs. 


\subsection{Northeast Region}

\subsubsection{Expert Keynote:}

\section{Mark Knight, Chief Engineer- Electricity Infrastructure Group, Pacific Northwest National Laboratory (PNNL)}

Mr. Knight's keynote presentation shared insights from his perspective working within the Grid Modernization Laboratory Consortia (GMLC) [9], which brings together leading experts, technologies, and resources at the U.S. Department of Energy's (DOE) national laboratories to collaborate on the goal of modernizing the nation's grid. More specifically, his focus is on transactive systems at PNNL. Interoperability is important for his work due to the sheer amount of intelligence appearing at the edge of the grid. If these domains and devices cannot communicate to each other, it will be problematic. Even while the focus of the industry is often on resilience, reducing downtime, and efficiency, if the cost of interoperability is reduced even a small amount, it can make a large overall impact. The wide impacts of interoperability are highlighted by the ten characteristics of a smart grid as described in EISA. All ten characteristics can be related to interoperability.

Interoperability is more complicated than simply plugging in devices and exchanging information. The GMLC published a white paper [10] on its interoperability strategic vision, which aims to "promote a common understanding of the meaning and characteristics of interoperability and to provide a strategy to advance the state of interoperability as applied to integration challenges facing grid modernization.” It defines interoperability as "the ability of two or more systems or components to exchange information and to use the information that has been exchanged." It is further described to include 3 categories, 8 layers, and multiple crosscutting issues, as shown in Figure 2.1 below.

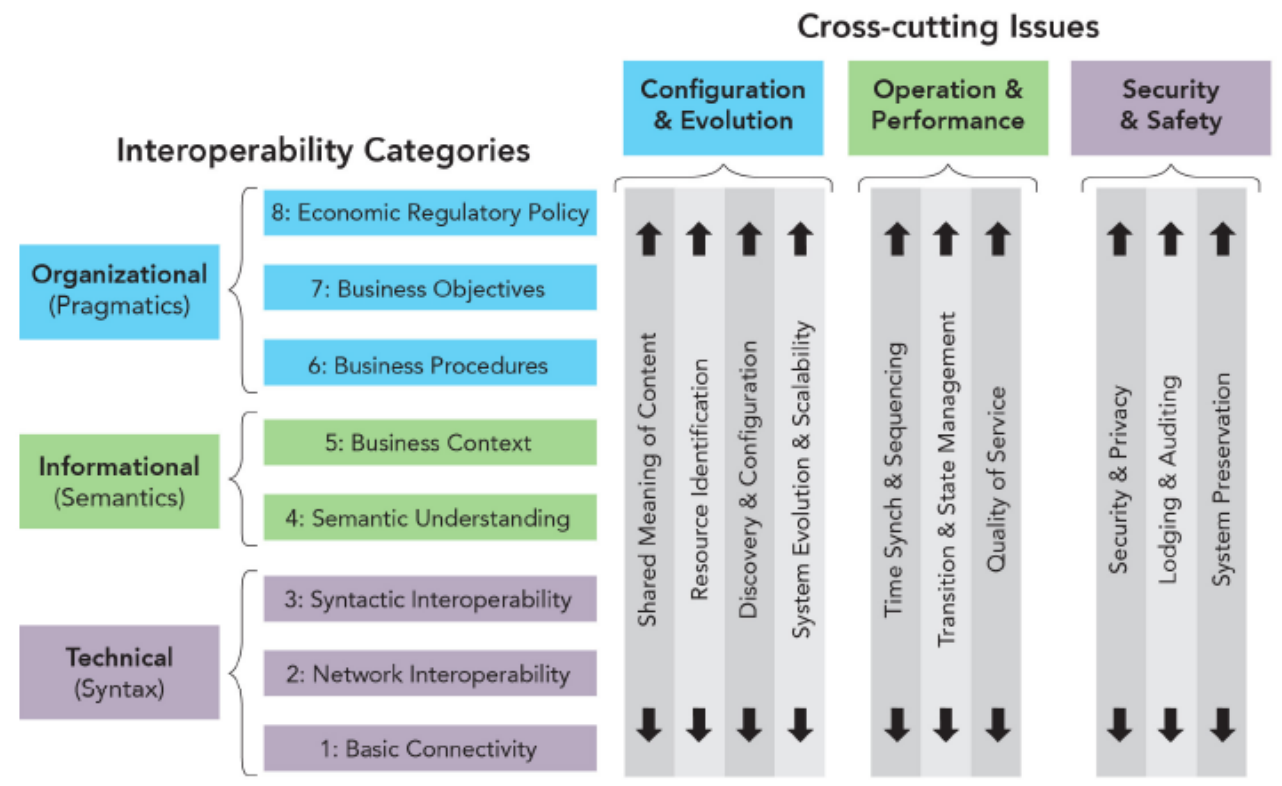

Figure 2.1 Proposed Schematic for Common Understanding of Interoperability 
The vision document focuses on the benefits of interoperability and the needs and role of the different stakeholders and sectors. The document also describes an interoperability maturity model that provides a measurement structure, using 35 criteria, to assess the state of interoperability in a specific technology deployment domain.

\subsubsection{Regulatory Keynotes:}

\section{Honorable Katie Dykes, Chair, Connecticut Public Utilities Regulatory Authority}

Over the past 20 years, accelerated by America Recovery and Reinvestment Act (ARRA) and EISA in the early 2000's, information technology has crept into all parts of the power sector, from power generation and transmission to smarter meters, smart appliances, and smart thermostats in homes and business. With these changes has come great potential to reduce costs, improve performance, and reduce risk. Rhode Island has passed a least cost procurement law to drive utility investments to benefit customers. However, the law raises a question on how to best evaluate the procurements. For example, some modernization investments come to the commission for review, but with lots of risk and uncertain conditions.

Interoperability within and across systems and technologies is an important hedge to avoid unnecessary sunk costs through obsolescence, however its quantified value is uncertain. It is not clear what questions regulators should be asking about interoperability when vetting proposals. For example, National Grid recently applied for funding to modernize its power sector, with proposed modernization objectives, distributed generation, electrification programs, and performance incentives. However, the Commission rejected the settlement agreement and proposed amendments related to power sector modernization. It is not clear if net benefits were calculated accurately, whether ratepayers would see the benefits, and whether they were correctly vetted based on the rapidly changing technology landscape. So, even though the Commission has adopted a modern, forward-looking rate design process, an improved approach to the evaluation of interoperability benefits is needed.

\section{Honorable Margaret Curran, Chairperson, Rhode Island Public Utilities Commission}

Commissions are not standards development organizations but should participate in their development in order to identify any specific concerns or questions and relate them back to NIST's Conceptual Diagrams. Workshops on these topics should be held regionally (as opposed to Washington, DC), since that is where the focus is. The Northeast region in particular would benefit from federal exchanges and working groups that are broader than a focus on individual states.

In Connecticut, there is a concern over the electrification of transportation due to uncertainty in the rate of customer adoption and how that impacts the level of investments that need to be made so the integration costs do not overwhelm any revenues from additional sales. Demand management will be fundamental to keep electricity prices stable while ensuring electrification. There is also concern over the functionality of Connecticut distribution utilities and what functionality they want to have. Regulators must think about grid 
investments in the context of the lifecycle, with investment plans taking into account the depreciation status of aging assets and replacement opportunities for fully depreciated assets. Once an investment is made, an important area that needs more focus is ensuring and verifying that the benefits do occur at a scale that is proportional with the utility investment. This information is required to determine the actual program performance and actual avoided costs. From there, the state and utility can determine what else needs to be done to maximize benefits.

Especially in the Northeast, which has six geographically small states, some of these issues should not be worked out in individual state hearing rooms. Rather, there could be benefits to a regional approach that brings utilities to the table and prevents the balkanization of New England's six markets. Another related challenge in the Northeast is with smaller, innovative service providers: they may not have a regulatory affairs staff person working with each Commission (i.e., for a small company it is difficult to interface with all six states in New England). So, it becomes difficult for one Commission to understand the industry's needs, especially on technical issues like interoperability. Another challenge is improving the PUCs' understanding of what to evaluate when vetting grid modernization proposals.

\subsubsection{Panel Discussion Highlights}

\section{Peter Kelly-Detwiler, Northbridge Energy Partners LLC}

Two key issues need to be addressed in the utility space - cybersecurity and resiliency.

Cybersecurity: With DER installations accelerating in the U.S. and around the world, the industry urgently needs to think about cybersecurity standards. There are vulnerabilities in the hardware that is being built_-for example, the German standards body TUV recently demonstrated that it could easily hack into commercially available inverters. There is a critical need to cyber-harden these systems as soon as possible, as hackers no longer need to even go through a SCADA system to attack anymore.

Resiliency: How will DERs contribute or harm grid resiliency? For example, EVs and storage are critical resiliency tools because they are designed to absorb and release large amounts of energy, but they can also be sources of vulnerabilities.

For both of these issues, Mr. Kelly-Detwiler suggested to start efforts with large assets first and then address smaller systems like batteries and EVs. He also emphasized that relevant parties must specify desired outcomes, not specific technology solutions.

\section{Matt Nelson, Massachusetts Department of Public Utilities}

A key theme in this topic is just how quickly things are moving due to technology development. Since technology development will not wait, there is a need to put frameworks in place that enable public policy goals, such as protecting consumers. A framework to 
consider while reviewing utility investment requests will aide regulators in responding more quickly, while also respecting the democratic process.

\section{Sidney "Macky" McCleary, Rhode Island Division of Public Utilities and Carriers}

The electric grid is fundamentally changing from one-way flow of electrons to a two-way flow of electrons and photons. With this change comes both risks and opportunities. With the legacy system, there was a central risk of failure. If interconnections are done properly, there is less overall risk of failure now. The role of the regulator is to facilitate this change to allow penetration of intelligence and flexibility into the grid, with corresponding benefits to the ratepayers over the long-term. What the regulator should not do is stop the market from adopting these new technologies. The challenge is how to provide important information to the grid operator, without increasing risks.

In addition to installing appropriate encryption and communication methods, interconnectivity standards need to be responsive and proactive to safeguard against threats. For example, standards can help with filtering data between the home and the utility. Lastly, with so many potential entry points, the detection of threats is most critical to ensure that as soon as a network is compromised, the threat is detected and can be neutralized before it causes major damage.

\section{Jim Perkinson, National Grid}

The "Golden Rule” with standards is to not depend on simulations solely, and bear in mind that the standard is only as good as the testing required to validate compliance. For example, in Germany a standard for inverters required self-certification, which ultimately did not show any improved performance due to the standard. Many companies do not think about how they can comply with a standard, but rather the minimum investment they can make to pass the test. For example, the CEC efficiency test that inverters need to meet to operate in California specifies testing at specific points, so companies would make inverters efficient only at those points. Standards landscape assessment graphics can be very helpful to understand the number of standards and corresponding tests.

\section{Henry Yoshimura, New England ISO (ISO-NE)}

DERs are currently around 16 percent of capacity in the New England states, and much of the new capacity being installed is DERs. ISO-NE peak load records were set back in the 2004 - 2006 timeframe, however there have been declines in both peak and total energy usage over time. On April 21, 2018, New England had to deal with the "Duck Curve," with its minimum midday load lower than the nighttime minimum. ISO-NE currently uses economic dispatch for DERs, without modeling the distribution system. However, in a high DER system, the physical capabilities of a distribution system could physically constrain the deployment and dispatch of DERs. Modeling of distribution systems is hard and complex, but granular models will eventually be needed. Distribution system operators may even be needed. 
Currently, the ISO operates a wholesale market, but there may be an increased focus on a more robust retail market. There will be a need for a type of operator to coordinate and determine the feasibility of DER markets and operations. Regulating trade between the ISO and DSO may also need to be determined as these shifts occur.

\section{Summary of Northeast Region Panel Q\&A}

The question and answer session included discussion on the following points:

- ISO-DSO architecture considerations: Work is ongoing on ISO-DSO interfaces in New England. As a simple comparison, New England utilities and providers offer services in six state jurisdictions where investor-owned utilities in California must comply with one state jurisdiction. Coordinating standards across jurisdictions may require additional time and monetary resource investment for either the jurisdictions or the utility, as compared to a single jurisdiction like California. There is not yet a clear framework or forum through which to address these types of issues (i.e., regional planning forums have yet to be leveraged in this context).

- $\quad$ Cybersecurity metrics and detecting cybersecurity threats: According to the NIST Cybersecurity Framework Version 1.1 [11] that identifies five cybersecurity functions (Identify, Protect, Detect, Respond, Recover), detection is not the first line of defense. However, detection is very important and new regulatory thinking will be needed in order to best reward and incentivize the detection of cybersecurity threats. One example of active research in this area is in physical unclonable functions (PUFs) where the actual physics of the system hardware is used as its unique fingerprint that cannot be faked. Along with this research, work needs to be done to identify an appropriate and measurable metric that can reward utilities to better detect threads.

Broader themes emphasized in the Eastern workshop discussions included the following:

- Seasonal challenges with gas delivery: In the Northeast, there are seasonal challenges associated with the gas delivery system. This regionally specific issue with New England's fierce winter includes complications associated with New England's market with summer and winter pricing. For example, when looking at peak time rebate benefit streams, a single time-based rate (TBR) is probably not the right approach since there are two different seasons with unique problem sets. There are other special New England winter-related challenges as well. While the DOE Quadrennial Energy Review looked at the relationship between electric and natural gas delivery systems in New England, it determined this is something that needs to be examined and addressed further at the customer end.

- Need for greater regional exchange of information: With its multiple small states, the New England region would benefit from information exchanges and working groups that are larger than individual states. This would help to avoid a balkanization of requirements, technologies, and markets. 


\section{Overarching Considerations for Smart Grid Interoperability Standards}

Discussions throughout the four regional workshops included a variety of overarching themes that were consistently brought up by participants. Technical (e.g., technological, measurement) as well as non-technical (e.g., economic, regulatory) issues were considered, highlighted below.

\section{Current and Future Issues for Interoperability Standards}

A number of broadly applicable national themes emerged as current and future issues for interoperability standards. Table 4.1 provides a summary of the issues that emerged as the most commonly discussed themes across the four workshops. The issues are separated into seven broad categories:
1) Operations
2) Economics
3) Cybersecurity
4) Reliability and Resilience
5) Testing and Certification
6) Standards Development
7) Non-Technical Challenges

These key issues, as well as other key overarching themes, are described in more detail below.

Table 33.1 Key Overarching Issues with Impacts on Interoperability Standards

\section{Operations \\ - Interoperability enables greater decentralized control of DERs \\ - Interoperability can help to accelerate electric vehicle (EV) adoption}

\section{Economics}

- Quantifying the benefits of interoperability is a challenge

- Interoperability is an important hedge against obsolescence

\section{Cybersecurity}

- A standardized risk framework is needed

- Desired cybersecurity outcomes should be specified in standards

\section{Reliability and Resilience}

- Interoperability contributes to robust resilience

- Ensuring reliability for high-DER scenarios is critical

\section{Testing and Certification}

- The continued need for robust testing infrastructure

- Determining appropriate interoperability profiles for test requirements is key 


\section{Standards Development}

- Nomenclature and ontology are challenges for standards development

- Running scenario analyses is important

\section{Non-Technical Challenges}

- Consumer engagement and education are essential

- Privacy and data-sharing issues must be resolved

\subsection{Operations}

Importance of interoperability for self-healing grids: Interoperability can help to enable self-healing grids, often facilitated by intelligent electronic devices (IEDs) such as smart switches, reclosers, sensors, and actuators that can re-route and restore power automatically if one part of the grid goes down (before the customer is even aware). Interoperability is vital in order to allow for utility distribution system equipment to communicate with meters, feeder equipment, substation equipment, and control system equipment that all may come from different vendors. The return on investment for self-healing has two main components. First, there are clear avoided O\&M costs as self-healing helps the utility identify which part of the system has gone down. Second, the capital investment in self-healing grid infrastructure pays for itself by avoiding outages, allowing the utility to maximize the rate base. However, despite the clear technology advantages, the current reliance on a manual (human) review and implementation of the self-healing recommendations may mean the full benefits of the interoperability are unrealized.

New types of resources are changing the operation of the grid: With the growth of wind and solar generation, storage, electric vehicles, and the expansion of energy efficiency and demand response resources, the way the electricity system is planned and operated is changing. This will alter the traditional ways the grid is organized, including moving away from capacity determinations or load following to supply following and better identification and utilization of resources at the distribution level.

Interoperability for optimized device communications: The use of the currently required communication protocols is limiting future functionality and the ability to have distributed intelligence. Federal and national agencies and organizations like NIST, DOE, and EPRI have an important role to play in providing guidance to states on device communication protocols.

Interoperability enables greater decentralized control of DERs: Interoperability standards may enable more decentralized control of DERs. Utilities need a level of trust and predictability from DERs, and the benefits of interoperability, in order to understand how those resources will affect the grid and ensure that the utility can maintain their services. Control and coordination of DER devices will enable the provision of grid services to customers, and the ability of customers to provide grid services. 
Importance of device proxies for high-DER scenarios: The high-DER scenario leads to a more meaningful interface between the customer domain and others, thereby elevating the importance of enabling devices to make decisions that reflect customer preferences in response to price signals. The communications pathway between the devices and the utility needs to be established, with devices acting as proxies for customers, including whether a third-party service provider can or is acting as an aggregator on behalf of customers.

Interoperability issues for microgrids: A challenge for microgrid integration is that the utility needs to know what it would have to supply if the entire microgrid generation went down - so the utility needs to know much, if not all, of what the microgrid knows. For shared assets like microgrids, the difficulty is that lines of accountability and responsibility are blurred. Risk assessments are needed, especially when the microgrid is large enough to impact the reliability of the wider area.

Microgrid control capabilities: The control capabilities of microgrids is dependent on a number of factors. For example, in times of emergencies like storm restoration, microgrid protocols should be able to communicate directly with a utility central dispatch. For utility managed microgrids, it also makes sense for the utility to have the capability to take control. The situation is not as clear for customer or third party owned microgrids where the level of control is dependent on customer expectations (i.e., does the customer expect the utility to back up the microgrid and perform tasks if it goes down?). Regardless of the situation, enhanced control capabilities are important for microgrids.

The priority microgrid interface is to the utility control center because that gives the microgrid the most visibility across the grid. It is not clear whether there is a difference between customer and utility managed microgrids related to that interface. Once there are enough microgrids, the utility can utilize regional microgrid controllers that may span large geographical areas. The interoperability profiles should be similar for microgrid controller vs. operations control center vs. utility controlled microgrid controlled vs. individual devices inside the microgrid. Even in cases where the utility does not control the microgrid (which are expected to number many times greater than utility-managed microgrids), communication is needed to ensure the microgrid adheres to the utility's rules. For example, depending on grid conditions, the utility may need to send a signal to the microgrid's generation resources to ramp up or down. Or in some cases the grid may be stable, but a signal needs to be sent to alter generation on a particular feeder.

Importance of preparing for emergency scenarios: A key gap of the NIST Interoperability Framework is its inability to deal with emergency scenarios. The Framework assumes everything is proceeding as expected, when in reality there will be unforeseen events that the Framework needs to be better able to address. For example, if a city center develops a microgrid, but across the street from a microgrid is an emergency shelter, then coordination is required to better understand how the shelter could make use of the microgrid. The technical requirements for "sunny day operations” vs. resilience (emergency) situations vs. other factors needs to be clarified. 
Rural vs. urban divide: A challenge for adoption of DERs is segmenting the dense urban areas vs. rural areas that are sparsely populated, but often are where the majority of generation is located. The development of microgrids in rural communities can help provide the benefits of interoperability to the rural community. Groups of co-ops, through economies of scale and improved bargaining power, may also be able to afford higher-density benefits to the rural communities.

Key interfaces for interoperability: The most important interfaces relevant for interoperability include electric vehicles, customer storage (batteries, water heaters), customer devices (including automation which enables the device to act as a proxy for a utility to human interface), and AMI information model. Coordination is needed in these interfaces that are witnessing a great deal of activity.

Importance of high-level diagrams to communicate the value of interoperability: Highlevel diagrams are useful for communicating with and educating electricity stakeholders to build broad understanding of the value of interoperability generally as well as specific technologies like AMI.

Interoperability can help to accelerate $\mathbf{E V}$ adoption: Increasing EV adoption can pose challenges to the grid. The mass deployment of these chargers would be an important opportunity for interoperability to relax.

Dispatchable Loads: Dispatchable loads (e.g., demand response) need special consideration as they can be used for many applications, including frequency response or to affect ramp rates. They can have a major impact on the required power, but appropriate communications and interoperability are needed.

Distinction between residential vs commercial/industrial interoperability: Residential and C\&I have different objectives, equipment, levels of sophistication, and priorities. Enterprise devices are much more important for C\&I, while residential is focused more on consumer devices that are more likely to be small, disposable, and have less security. Interoperability between the two should be separated.

Interoperability to assist in cultivating trust: Trustworthiness is an area of growing concern that needs to be addressed. There is a need for governance structures to better cultivate trust between utilities and third parties. The presence of split incentives on who is responsible/liable makes trust difficult to achieve. The development of appropriate algorithms may be needed.

\subsection{Economics}

Quantifying the benefits of interoperability is a challenge: This was a consistent theme throughout all workshops. Utilities need to be able to develop cost-benefit analyses and make the business case for investments. However, the inability to communicate the business case of interoperability and adequately quantify the benefits of interoperability, especially when there are long-term benefits and unanticipated benefits, is a challenge. Value metrics for 
interoperability and quantification tools, especially related to AMI, are needed. It is important to measure and document the benefits of interoperability after the fact-in order to help develop the business case for future projects. Most unanticipated benefits tend to favor the customer in the form of operational efficiencies that flow to customer in the form of reduced electricity bills and other economic opportunity. It is also important to note that benefits will be highly dependent on specific policy considerations. An example is feeder automation; in the US, every dollar invested in feeder automation resulted in five dollars of return. There was no business case in the UK for the same technology because fault detection and volt-var control were required already by policy.

EPRI published a report in 1984 that detailed the hard-to-identify benefits of distribution automation (DA) [12]. This report, which collected all the benefits in one document, became a critical resource for anyone who needed to argue the benefits of DA. An analogous type of report for interoperability could offer similar benefits.

Unanticipated benefits of interoperability: There is a need for the industry to better convey the benefits of interoperability to the customer, including potentially unanticipated benefits and benefits to those stakeholders who most fear being disadvantaged.

The benefits of advanced technology need to be better defined and communicated: Utilities are struggling with communicating the benefits of new technology deployments. Operational benefits that result in improved services to customers (e.g., avoided outages) are not being communicated clearly to customers and regulators. Typically, utilities only submit identified known benefits, and do not identify potential or soft benefits. As a result, the only benefits some consumers associate with an investment are those that they can see. As discussed during the workshops, AMI is an example of an investment that has resulted in lots of avoided costs, as opposed to explicit new benefits, but neither of which were explicitly accounted for in many business cases around the country.

Interoperability is an important hedge against obsolescence: Interoperability is an important hedge against obsolescence, but the quantified value of that hedge is uncertain. An improved approach is needed. Interoperability will help to manage legacy hardware through planned obsolescence.

Multi-vendor procurements: Interoperability will allow for multi-vendor procurements for capital equipment that are all compatible with a particular standard. If a particular company goes out of business, then the products can still be managed if they follow the appropriate standard.

Interoperability and procurement: Best practice in the software world is to develop and release a system, with the expectation that it will then be refined and updated over time. This kind of capability is missing from much grid hardware, so there is a risk of installing a system that then gets outdated before it is even implemented. Utilities need to have hardware specified by multiple departments at the development stage, but the hardware needs to have the capability to support software refinements over time. In other words, with costs of hardware dropping, there should be ways to deploy systems that can be easily updated to 
keep up with the rapidly changing grid space. Thus, with interoperability, utilities will be better able to procure systems with greater functionality, as long as they meet the appropriate interoperability standard.

Evaluation of proposals: Regulators need guidance on the types of questions that should be asked when reviewing proposals for smart grid devices that should include interoperability.

Making the business case for charging station installation: It's difficult to quantify the future benefits of EV charging station installation, taking into account the needs of the driver and utility.

Unlocking creative uses of new investments: New technologies are generating a lot of data and utilities and third parties are learning how to make use of that data beyond what was expected. However, existing mandates and requirements may limit the use of that data. For example, some utilities are limited in utilizing the full benefits of AMI—such as using AMI voltage data to inform on fault indication-due to NERC critical infrastructure protection (CIP) requirements. Co-operatives, which are generally not subject to NERC CIP requirements, are able to make more creative use of AMI.

Impact of interoperability on customers: There is a lack of customer incentives for interoperability, even though a good portion of the benefits for interoperability is to empower customers by seeking and obtaining additional benefits from assets. The role of the customer is becoming more important, with the grid becoming more and more decentralized with intelligence embedded along the grid edge. For example, in places like Hawaii, distributed resources may soon be larger than central resources.

Importance of data analytics: Meters are the foundational hardware, but all other benefits are enabled by powerful data analytics and integrated technology solutions.

Interoperability is key to value stacking: Interoperability enables easier communications among various technologies, which can be critical for value stacking applications. For example, if smart switches can coordinate among all the different water heaters in a neighborhood, value can be derived by aggregating or coordinating those heaters in response to a price or other signal. If the water heaters have poor interoperability, the ability to coordinate response is limited, and, thus, the value to the grid is greatly diminished.

\subsection{Cybersecurity}

Standardized risk framework: The standardization of security includes interoperability. There needs to be a standardized risk framework to assess risk.

Desired cybersecurity outcomes should be specified in standards: For cybersecurity standards, it is important to specify outcomes rather than specific technologies. The NIST Cybersecurity Framework and Smart Grid Profile is good start, but more needs to be done. 
Brokerless communications security: It is not clear how to secure brokerless communication protocols.

\subsection{Reliability and Resiliency}

Interoperability contributes to robust resiliency: Resiliency is critical in addressing grid failures, which need to be expected as a normal part of operations for large systems of systems. Having in place the appropriate interoperability standards is an important step to ensuring robust resiliency.

Ensuring reliability for high-DER scenarios is critical: For high-DER penetration scenarios, utilities need to have visibility behind the meter or see a very reliable model for it at the point of interconnection to ensure that power supply can be maintained if solar suddenly drops. For example, this can be particularly important in residential neighborhoods with high levels of solar PV penetration. There are lessons learned that should be examined from European experience (e.g., Denmark and Germany) with high penetration of renewables.

Reliability standards: Need to move from reliability (security of supply) to hosting capacity over time.

Accelerating smart grid technology adoption through reduced risk: The deployment of smart grid technologies can be inhibited by utilities' desire to minimize risk. In addition to developing the technology, mitigating these risks must be taken into account in order to successfully develop interoperability standards. These risks include grid security and the possibility of bad actors attempting to disrupt or destroy grid operations. Interoperability standards play an important role in the acceptance and deployment of smart grid technologies. Incentives, legislations, and economics also play important roles.

Improved modeling: Current grid modeling can show stable results given an initial state when run on one platform, but on another platform can conclude the same initial state results in unstable conditions. Uncertainty in measurements like frequency could influence such dichotomy. Similarly, issues with measurement of frequency have caused issues in the grid related to solar inverters.

\subsection{Testing and Certification}

Importance of robust testing: A standard is only as good as the test it is verified against. So, it is important to provide better described interoperability requirements, which would then lead to improved testing.

Determining appropriate interoperability profiles to test requirements: It is important to work with partners to better determine which interoperability profiles are most important and use these profiles to test requirements. Some of the key interfaces where interoperability profiles may be most useful include 1) EVs and customer-sited storage, 2) AMI information 
models, and, 3) Customer devices and customer device automation as a proxy for the interface.

Certification and innovation: Concern that standards and certification could inhibit innovation because the products will be designed to meet the lowest bar. Need to ensure standards being developed will not be a detriment to innovation. However, an advantage of certification is that a third party is doing the testing, which provides greater assurance to regulators.

\subsection{Standards Development}

Nomenclature and ontology are challenges for standards development: Appropriate nomenclature is needed to enable intelligent discussions that span several distinct communities. These communities each have their own nomenclature, so the challenge is to respect all the disciplines, but still find a way to integrate where appropriate. For example, the use of certain languages targeted to specific audiences can lose other important audiences in the process. In some case there is a need for organizations to create new terms, while in other cases organizations have already developed a rich language to communicate technically_and reference will need to be made to their work.

With this type of an interdisciplinary issue, it is important to have a common understanding of not only nomenclature, but also the problem that the standard is trying to solve (e.g., inequitable costs for regulators, utilities-cost for recovery, customers, system engineers, etc.). This is especially important given that stakeholders may have conflicting priorities on timelines and business processes. Defining appropriate problem statements help to engage stakeholders on the conversation for why a particular standard is important to implement and beneficial over the long term, despite it being difficult to implement in the short term.

Importance of running scenario analyses: In parallel with the development of a standard, it is critical to run planning exercises such as scenario analyses and have contingencies in place to account for changes to the market or grid that take place during standards development. For example, if the standard being developed does not take into account utilities' business model reform or accelerating deployment of DER, then it may not be successfully developed and adopted by the market. Assumptions may need to be questioned to take into account radical changes that may come into effect, for example, allowing for the emergence of active consumers that can buy or sell energy directly to each other or future load curves that will look like flat horizons.

Ensure no overlapping standards: Once overlapping standards reach the market, it is a major challenge to harmonize and integrate-in some cases it may not even be possible. So, focus is needed to ensure no overlapping standards reach the market in the first place.

Fill in gaps where standards are missing: In some areas there are an abundance of standards, while in others there are gaps (e.g., management infrastructure) that are in need of standards. 
Open standards: Open standards are important because they enable interoperability. They need to be collaborative, open and transparent, and published to the public domain with reasonable fees. Examples include ANSI C12.22 for meters and OpenADR for load shedding.

Mismatch of lifecycles: Some utility infrastructure hardware has very long lifetimes, but software may have shorter lifespans. Furthermore, many consumer devices have lifetimes of 1-2 years, meaning the consumer devices may change over 15 times over the course of the utility infrastructure. This needs to be taken into account when developing related standards and policies.

Increase the speed of standards development: Standards typically take years to develop, which all stakeholders agree is too long. Effort needs to be made to reduce the time it takes to develop a new standard. For example, the time it takes for technology companies to develop software is on the scale of weeks. Is there anything that can be learned from the software development process?

Interoperability conformance: The steps to achieve interoperability need to be defined. Conformance to a standard does not achieve interoperability because standards often include sufficient flexibility in their application, or conformance may be limited to only certain parts of the standard. Ensuring interoperability will likely require defining a narrower scope built upon a standard that describes the capabilities, functionality, protocols, and data models that will support the application.

Marketing of standards: Some technology companies that are not fully aware of the standards that are applicable to their products. A better way to inform companies about applicable standards is needed so the companies do not have to perform a literature review on their own.

\subsection{Non-Technical Challenges}

Importance of consumer and regulatory engagement and education: Consumer engagement and education needs to be at the forefront in order to help customers and regulators better understand and trust interoperability.

Role of the regulator: With increasingly technical investments being proposed by utilities, regulators need more support, educational opportunities, and access to experts to assist them in reviewing utility applications. Regulators will also be increasingly put into a position of overseeing utility plans, applications, and proposals that require a better understanding of interoperability as it applies to the utility, utilities’ vendors, customers, and other third parties.

Local optimization: What does interoperability look like when optimization becomes more local? NIST does have a research program addressing local optimization. 
Rapid speed of change: Smart grid technology development and deployment is accelerating. Do voluntary standards play a role in accelerating the acceptance of new technologies or can voluntary standards play a role in innovation?

Third party integrators: There is currently no demand for alternatives to the third-party integrator model because it is the way it has always been done. But greater migration of data to the cloud may force a breaking point leading to a new model other than third party integrators.

Data sharing and privacy considerations: Privacy is a major issue with customers, with still unanswered questions around the topics of to what extent do utilities need customer info and the role of the utility in privacy considerations.

\section{Feedback on NIST interoperability framework:}

- Value of the Framework should not be to show the answers to interoperability because that becomes dated immediately. Instead its best used as a tool to help understand progress with respect to grid modernization, the current direction, and help to identify gaps to getting to the modern grid.

- How will EV, which is a mobile energy storage device, be considered as a conceptual model and domain in the Framework Version 4.0?

- How should demand response aggregators be considered in the diagram? NIST needs to determine the best way to include DR in the generation domain.

- Universities are absent on diagram.

\section{Next Steps}

This report summarizes the key regional themes on smart grid interoperability elicited from stakeholders attending a series of roundtable meetings across the United States. The report will be used to provide inputs for updates of the Framework.

NIST is working on a major new release of the Framework. NIST has engaged stakeholders in industry, government, and academia for advice and feedback during the Framework development process. This outreach includes multiple workshops in addition to the roundtables that were attended by a range of smart grid stakeholders, from utilities, vendors, and equipment manufacturers to renewable power producers, retail service providers, and regulators. Workshop sessions were used to gain feedback as well as help stakeholders understand the role interoperability will have in shaping today's complex electricity ecosystem.

NIST continues to seek information to improve the value and usefulness of the Framework. NIST is also conducting a full cycle of research, development, and innovation to provide the foundations for the future grid. The NIST Smart Grid program advances the measurement science necessary to increase asset utilization and efficiency, improve grid reliability, and enable greater use of renewable energy sources in the grid through research, standardization, testing and implementation of the NIST Framework. 


\section{Acknowledgement}

This report is based on the results of four regional workshops held across the United States. The workshops were conducted by the National Institute of Standards and Technology (NIST) in partnership with the National Association of Regulatory Utility Commissioners (NARUC).

Many thanks are extended to the NIST organizers and public utility commissions in various states: Georgia Public Service Commission, Indiana Utility Regulatory Commission, California Public Utilities Commission, and the Rhode Island Public Utilities Commission. The regional and national perspectives provided by keynote and plenary speakers and panelists were highly valuable in setting the stage for discussions. We also appreciate the extensive contributions of the participants; this report would not be possible without their valuable insights. Finally, thanks are extended to Energetics for assistance in the preparation of this report. 


\section{References}

[1] Arnold, G. W., Wollman, D. A., FitzPatrick, G. J., Prochaska, D., Holmberg, D. G., Su, D. H., Hefner Jr., A. R., Golmie, N. T., Brewer, T. L., Bello, M., \& Boynton, P. A. (2010). NIST Framework and Roadmap for Smart Grid Interoperability Standards, Release 1.0 (NIST-SP-1108). DOI: 10.6028/NIST.sp.1108.

[2] Energy Independence and Security Act of 2007 [Public Law No: 110-140].

[3] Arnold, G., FitzPatrick, G., Wollman, D., Nelson, L., Boynton, P., Koepke, G., Hefner Jr., A., Nguyen, C., Mazer, J., Prochaska, D., Swanson, M., Brewer, T., Pillitteri, V., Su, D., Golmie, N., Simmon, E., Eustis, A., Holmberg, D., Bushby, S., Janezic, M., \& Jillavenkatesa, A. (2012). NIST Framework and Roadmap for Smart Grid Interoperability Standards, Release 2.0. (NIST-SP-1108R2). DOI: 10.6028/NIST.sp.1108r2.

[4] Greer, C., Wollman, D., Prochaska, D., Boynton, P., Mazer, J., Nguyen, C., FitzPatrick, G., Nelson, T., Koepke, G., Hefner Jr., A., Pillitteri, V., Brewer, T., Golmie, N., Su, D., Eustis, A., Holmberg, D., \& Bushby, S. (2014). NIST Framework and Roadmap for Smart Grid Interoperability Standards, Release 3.0. (NIST SP1108r3), DOI: 10.6028/NIST.SP.1108r3.

[5] Marron, J., Gopstein, A., Bartol, N., \& Feldman, L. (2019). Cybersecurity Framework Smart Grid Profile (NIST-TN-2051). DOI: 10.6028/NIST.TN.2051.

[6] Institute of Electrical and Electronics Engineers. (2018). IEEE Standard for Interconnection and Interoperability of Distributed Energy Resources with Associated Electric Power Systems Interfaces (IEEE Std 1547-2018). DOI: 10.1109/IEEESTD.2018.8332112.

[7] International Electrotechnical Commission Common Information Model (CIM) standards IEC 61970 (Energy Management) and IEC 61968 (Distribution Management). https://www.iec.ch/smartgrid/standards/.

[8] Trabish, H. (2016). Inside the first municipal solar-plus-storage project in the US. Utility Dive. https://www.utilitydive.com/news/inside-the-first-municipal-solar-plusstorage-project-in-the-us/421470/.

[9] Department of Energy Grid Modernization Lab Consortium. https://www.energy.gov/grid-modernization-initiative-0/grid-modernization-labconsortium

[10] Department of Energy Grid Modernization Lab Consortium (2018). Interoperability Strategic Vision: A GMLC White Paper. https://gmlc.doe.gov/sites/default/files/resources/InteropStrategicVisionPaper201803-29.pdf

[11] National Institute of Standards and Technology (2018). Framework for Improving Critical Infrastructure Cybersecurity Version 1.1. DOI: 10.6028/NIST.CSWP.04162018

[12] Bunch, J. (1984). Guidelines for evaluating distribution automation. EPRI Report EL-3728. 


\section{Appendix A: Participants}

\section{Southeast Region}

\begin{tabular}{|l|l|}
\multicolumn{2}{|c|}{ Name } \\
\hline Bob Bergstrom & Copper Tree \\
\hline Steven Pullins & Dynamic Energy Networks \\
\hline Scott Morgan & Energetics \\
\hline Aaron Snyder & EnerNex \\
\hline Rick Wornat & EnerNex \\
\hline Donald Polmann & Florida Public Service Commission \\
\hline John McDonald & GE Grid Solutions \\
\hline Jamie Barber & Georgia Public Service Commission \\
\hline Nick Cooper & Georgia Public Service Commission \\
\hline Ben Deitchman & Georgia Public Service Commission \\
\hline William Edge & Georgia Public Service Commission \\
\hline Deborah Flannagan & Georgia Public Service Commission \\
\hline Nicholas Hall & Georgia Public Service Commission \\
\hline John Kaduk & Georgia Public Service Commission \\
\hline Sheree Kernizan & Georgia Public Service Commission \\
\hline Lauren McDonald & Georgia Public Service Commission \\
\hline Rob Trokey & Georgia Public Service Commission \\
\hline Jason Allnutt & IEEE - Standards Association \\
\hline Steve Chasu & Landis \& Gyr \\
\hline Andrew Marshall & Landis \& Gyr \\
\hline & $\begin{array}{l}\text { National Association of Regulatory Utility Commissioners } \\
\text { (NARUC) }\end{array}$ \\
\hline Kerry Worthington & National Institute of Standards and Technology (NIST) \\
\hline Avi Gopstein & National Institute of Standards and Technology (NIST) \\
\hline Cheyney O'Fallon & National Institute of Standards and Technology (NIST) \\
\hline Paul Boynton & North American Electric Reliability Corporation (NERC) \\
\hline Fritz Hirst & North American Electric Reliability Corporation (NERC) \\
\hline Mark Lauby & pdvWireless \\
\hline Robert Burkhardt & Plugged In Strategies \\
\hline Chris Villarreal & Southern Company \\
\hline Katherine Prewitt & Southern Company Services \\
\hline Howard Smith & UL LLC \\
\hline Bill Colavecchio & Wedin Communications LLC \\
\hline Randy Wedin & \\
\hline
\end{tabular}




\section{Midwest Region}

\begin{tabular}{|l|l|}
\multicolumn{2}{|c|}{ Name Affiliation } \\
\hline Russell De Salvo & ComEd \\
\hline Scott Morgan & Energetics \\
\hline Wanda Reder & Grid-X Partners \\
\hline Pam Johnson & Hosted by MN PUC \\
\hline Ravi Subramaniam & IEEE Standards Association \\
\hline Tomas Rodriguez & Illinois Commerce Commission \\
\hline Tanner Guthrie & Indiana Michigan Power Cmpany \\
\hline Steve Davies & Indiana Utility Regulatory Commission \\
\hline Sarah Freeman & Indiana Utility Regulatory Commission \\
\hline David Johnston & Indiana Utility Regulatory Commission \\
\hline Edward Eckert & Itron, Inc. \\
\hline Jeremy Comeau & Indiana Utility Regulatory Commission \\
\hline Brad Pope & Indiana Utility Regulatory Commission \\
\hline Eileen Hardy & Kentucky Energy Cabinet \\
\hline Kenya Stump & Kentucky Office of Energy Policy \\
\hline Lynne Kiesling & Knowledge Problem \\
\hline Lance Mabry & Law Office \\
\hline Gregory Ehrendreich & Midwest Energy Efficiency Alliance (MEEA) \\
\hline Danielle Sass Byrnett & $\begin{array}{l}\text { National Association of Regulatory Utility Commissioners } \\
\text { (NARUC) }\end{array}$ \\
\hline Paul Boynton & National Institute of Standards and Technology (NIST) \\
\hline Avi Gopstein & National Institute of Standards and Technology (NIST) \\
\hline Cheyney O'Fallon & National Institute of Standards and Technology (NIST) \\
\hline Robert Burkhardt & PDV Wireless \\
\hline Christopher Villarreal & Plugged In Strategies \\
\hline Gary England & UltraSmart Controls \\
\hline John Donleavy & Utilligent \\
\hline Craig Pennington & Wabash Valley Power Association \\
\hline Christopher Stokes-Pham & Wabash Valley Power Association \\
\hline & \\
\hline
\end{tabular}


Western Region

\begin{tabular}{|l|l|}
\multicolumn{1}{|c|}{ Name } & \multicolumn{1}{c|}{ Affiliation } \\
\hline Thomas Lee & AutoGrid Systems \\
\hline Jose Aliaga-Caro & California Public Utilities Commission \\
\hline Wendy Al-Mukdad & California Public Utilities Commission \\
\hline Danjel Bout & California Public Utilities Commission \\
\hline Brian Korpics & California Public Utilities Commission \\
\hline Sophie Meyer & California Public Utilities Commission \\
\hline Rajan Mutialu & California Public Utilities Commission \\
\hline Carla Peterman & California Public Utilities Commission \\
\hline Ehren Seybert & California Public Utilities Commission \\
\hline Steven Ray & Carnegie Mellon University \\
\hline Ane Smart & ChargePoint \\
\hline Jeff Ackerman & Colorado Public Utilities Commission \\
\hline Candace Suh-Lee & Electric Power Research Institute (EPRI) \\
\hline Don Von Dollen & Electric Power Research Institute (EPRI) \\
\hline Lorenzo Kristov & Electric System Policy \\
\hline Caroline Dollinger & Energetics \\
\hline Kavya Balaraman & Energy Newsdata \\
\hline James Fine & Environmental Defense Fund \\
\hline Ravi Subramanian & IEEE - Standards Association \\
\hline Kerry Worthington & $\begin{array}{l}\text { National Association of Regulatory Utility Commissioners } \\
\text { (NARUC) }\end{array}$ \\
\hline Avi Gopstein & National Institute of Standards and Technology (NIST) \\
\hline Chris Greer & National Institute of Standards and Technology (NIST) \\
\hline Cuong Nguyen & National Institute of Standards and Technology (NIST) \\
\hline Cheyney O'Fallon & National Institute of Standards and Technology (NIST) \\
\hline Farrokh Rahimi & Open Access Technology International, Inc (OATI) \\
\hline Roy Lum & Pacific Gas and Electric (PG\&E) \\
\hline Arvind Simhadri & Pacific Gas and Electric (PG\&E) \\
\hline Robert Burkhardt & pdvWireless \\
\hline Tanya Barham & PECI \\
\hline Christopher Villarreal & Plugged In Strategies \\
\hline Steve Shoemaker & Public Advocats Office \\
\hline Adam Danise & Public Utilities Commission of Nevada (PUCN) \\
\hline Joseph Hughes & Reef Energy Systems \\
\hline Poormehr Honarmand & Sunrun \\
\hline Ann Rendahl & Washington Utilities and Transporation Commission (WUTC) \\
\hline Kathi Scanlan & Washington Utilities and Transporation Commission (WUTC) \\
\hline
\end{tabular}




\section{Northeast Region}

\begin{tabular}{|c|c|}
\hline Name & Affiliation \\
\hline Amy Boyd & Acadia Center \\
\hline Hank Webster & Acadia Center \\
\hline Katie Dykes & Connecticut Public Utilities Regulatory Authority \\
\hline Caroline Dollinger & Energetics \\
\hline Jason Allnutt & IEEE - Standards Association \\
\hline Stephanie Pine & Intelligent Power and Energy Research Corporation (IPERC) \\
\hline Henry Yoshimura & ISO New England \\
\hline Weezie Nuara & ISO New England \\
\hline Matthew Nelson & Massachusetts Department of Public Utilities \\
\hline James Perkinson & National Grid \\
\hline Avi Gopstein & National Institute of Standards and Technology (NIST) \\
\hline Cheyney O'Fallon & National Institute of Standards and Technology (NIST) \\
\hline Cuong Nguyen & National Institute of Standards and Technology (NIST) \\
\hline Rachel Goldwasser & $\begin{array}{l}\text { New England Conference of Public Utilities Commissioners } \\
\text { (NECPUC) }\end{array}$ \\
\hline Ben D'Antonio & New England States Committee on Electricity (NESCOE) \\
\hline Doug Sabetti & Newport Solar \\
\hline Patricia Fillipino & Newport Solar \\
\hline Kurt Demmer & NH Public Utilities Commission \\
\hline Peter Kelly-Detwiler & Northbridge \\
\hline Mike Guerard & Optimal Energy \\
\hline Sam Ross & Optimal Energy \\
\hline Mark Knight & Pacific Northwest National Laboratory (PNNL) \\
\hline Robert Burkhardt & pdvWireless \\
\hline Christopher Villarreal & Plugged In Strategies \\
\hline Abigail Anthony & Rhode Island Public Utilities Commission \\
\hline Margaret Curran & Rhode Island Public Utilities Commission \\
\hline Patti Lucarelli & Rhode Island Public Utilities Commission \\
\hline Todd Bianco & Rhode Island Public Utilities Commission \\
\hline Marion Gold & State of Rhode Island \\
\hline Taylor Spait & Utilidata \\
\hline
\end{tabular}




\section{Appendix B: Regional Workshop Agendas}

This appendix shows the agendas of the four regional roundtable meetings that were held to discuss revisions and updates to the NIST Smart Grid Interoperability Framework. All meetings were co-hosted by NIST and the National Association of Regulatory Utility Commissioners (NARUC).

Southeast Region: September 12, 2018, in Atlanta, Georgia

\begin{tabular}{|c|c|}
\hline \multicolumn{2}{|r|}{$\begin{array}{l}\text { Southeast Regional Roundtable: } \\
\text { Framework and Roadmap of Smart Grid Interoperability Standards } \\
\text { Georgia Public Service Commission } \\
244 \text { Washington Street, Atlanta, Georgia } 30334\end{array}$} \\
\hline 9:00 am & $\begin{array}{l}\text { Welcome AND AGENDA } \\
\text { - Honorable Lauren "Bubba" McDonald, Chairman, Georgia Public Service Commission }\end{array}$ \\
\hline 9:30 am & $\begin{array}{l}\text { KEYNOTE } \\
\text { - Mark Lauby, Senior Vice President \& Chief Reliability Officer, North American Electric Reliability Corporation }\end{array}$ \\
\hline $10: 15 \mathrm{am}$ & BREAK \\
\hline $10: 30 \mathrm{am}$ & $\begin{array}{l}\text { PRIMER ON NIST INTEROPERABILITY FRAMEWORK AND SMART GRID CONCEPTUAL MODELS } \\
\text { - } \quad \text { Avi Gopstein, NIST }\end{array}$ \\
\hline $11: 15 \mathrm{am}$ & $\begin{array}{l}\text { FACILITATED DISCUSSION } \\
\text { - Paul Boynton and Avi Gopstein, NIST. Participants discuss key questions about the representative grid } \\
\text { "architecture" and local priorities. }\end{array}$ \\
\hline $12: 15$ pm & LUNCH BREAK (ON YOUR OWN) \\
\hline $\mathrm{I}: 30 \mathrm{pm}$ & $\begin{array}{l}\text { REVIEW AND UPDATE ON IEEE } 1547 \\
\text { - } \quad \text { Ravi Subramaniam, IEEE Standards Association }\end{array}$ \\
\hline $\mathrm{I}: 45 \mathrm{pm}$ & $\begin{array}{l}\text { PERSPECTIVES ON THE BENEFITS OF SMART GRID INTEROPERABILITY } \\
\text { Panelists: } \\
\text { - Jamie Barber, Georgia Public Service Commission } \\
\text { - Bill Colavecchio, Underwriters Laboratories } \\
\text { - John McDonald, General Electric Grid Solutions } \\
\text { - Howard Smith, Southern Company } \\
\text { - Moderator: Chris Villarreal, Plugged In Strategies }\end{array}$ \\
\hline 2:45 pm & BREAK \\
\hline $3: 00$ pm & $\begin{array}{l}\text { FACILITATED DISCUSSION: CAPTURING PARTICIPANT INSIGHTS INTO GRID OPERATIONS AND } \\
\text { ECONOMICS } \\
\text { - How does interoperability relate to local operations? } \\
\text { - What are the constraints that limit asset utilization? } \\
\text { - How can interoperability improve return on investment? } \\
\text { - What steps can be taken to maximize device and infrastructure usefulness over its } \\
\text { physical lifetime? }\end{array}$ \\
\hline 4:00 pm & ConCLUSION AND NEXT StePS \\
\hline $4: 30 \mathrm{pm}$ & ADJOURN \\
\hline
\end{tabular}


Midwest Region: September 27, 2018, in Indianapolis, Indiana

\begin{tabular}{|c|c|}
\hline$F$ & $\begin{array}{l}\text { Midwest Regional Roundtable: } \\
\text { amework and Roadmap of Smart Grid Interoperability Standards } \\
\text { Indiana Utility Regulatory Commission } \\
\text { I0I W. Washington Street, Suite I500E, Indianapolis, IN } 46204\end{array}$ \\
\hline $9: 00 \mathrm{am}$ & $\begin{array}{l}\text { Welcome AND AGENDA } \\
\text { - Honorable Sarah Freeman, Commissioner, Indiana Utility Regulatory Commission }\end{array}$ \\
\hline $9: 30 \mathrm{am}$ & $\begin{array}{l}\text { KEYNOTE } \\
\text { - Wanda Reder, President and Chief Executive Officer, Grid-X Partners }\end{array}$ \\
\hline $10: 15 \mathrm{am}$ & BREAK \\
\hline $10: 30 \mathrm{am}$ & $\begin{array}{l}\text { REgULATION IN FocUS: THE VIEW OF THE REGULATOR } \\
\text { - Honorable Sarah Freeman, Commissioner, Indiana Utility Regulatory Commission }\end{array}$ \\
\hline I I:00 am & $\begin{array}{l}\text { PRIMER ON NIST SMART GRID CONCEPTUAL MODELS } \\
\text { - } \quad \text { Avi Gopstein, NIST }\end{array}$ \\
\hline $\mathrm{II}: 30 \mathrm{am}$ & $\begin{array}{l}\text { FACILITATED DiscuSSION } \\
\text { - Participants discuss key questions about the representative grid "architecture" and local priorities. }\end{array}$ \\
\hline $12: 30$ pm & LUNCH BREAK (ON YOUR OWN) \\
\hline $\mathrm{I}: 30 \mathrm{pm}$ & $\begin{array}{l}\text { PERSPECTIVES ON THE BENEFITS OF SMART GRID INTEROPERABILITY } \\
\text { Panelists: } \\
\text { - } \quad \text { Russ Desalvo, Commonwealth Edison } \\
\text { - } \quad \text { Ed Eckert, Itron } \\
\text { - } \quad \text { Lynne Kiesling, Purdue University } \\
\text { - } \quad \text { Kenya Stump, Kentucky Office of Energy Policy } \\
\text { - } \quad \text { Moderator: Chris Villarreal, Plugged In Strategies }\end{array}$ \\
\hline $2: 30 \mathrm{pm}$ & BREAK \\
\hline $2: 45 \mathrm{pm}$ & $\begin{array}{l}\text { FACILITATED DISCUSSION: CAPTURING PARTICIPANT INSIGHTS INTO GRID OPERATIONS AND } \\
\text { ECONOMICS } \\
\text { - How does interoperability relate to local operations? } \\
\text { - What are the constraints that limit asset utilization? } \\
\text { - How can interoperability improve return on investment? } \\
\text { - What steps can be taken to maximize device and infrastructure usefulness over its } \\
\text { physical lifetime? }\end{array}$ \\
\hline $3: 45$ pm & $\begin{array}{l}\text { REVIEW AND UPDATE ON IEEE } 1547 \\
\text { - } \quad \text { Ravi Subramaniam, IEEE Standards Association }\end{array}$ \\
\hline $4: 00 \mathrm{pm}$ & CONClusion AND Next Steps \\
\hline $4: 30 \mathrm{pm}$ & ADJOURN \\
\hline
\end{tabular}


Western Region: October 16, 2018, in San Francisco, California

\begin{tabular}{|c|c|}
\hline \multicolumn{2}{|c|}{$\begin{array}{l}\text { Western Regional Roundtable: } \\
\text { Framework and Roadmap of Smart Grid Interoperability Standards }\end{array}$} \\
\hline 9:00 am & $\begin{array}{l}\text { Welcome and Agenda } \\
\text { - Honorable Carla Peterman, Commissioner, California Public Utilities Commission }\end{array}$ \\
\hline $9: 30 \mathrm{am}$ & $\begin{array}{l}\text { KEYNOTE } \\
\text { - Don Von Dollen, Senior Program Manager, Electric Power Research Institute }\end{array}$ \\
\hline $10: 15$ am & BREAK \\
\hline $10: 30 \mathrm{am}$ & $\begin{array}{l}\text { REgULATION IN FocuS: THE VieW OF THE REgULATOR } \\
\text { - Honorable Jeffrey Ackermann, Chairman, Colorado Public Utilities Commission }\end{array}$ \\
\hline II:00 am & AUDIENCE POLL: KEY INTEROPERABILITY ISSUES \\
\hline $11: 15$ am & $\begin{array}{l}\text { PRIMER ON NIST SMART GRID CONCEPTUAL MODELS } \\
\text { - } \quad \text { Avi Gopstein, NIST }\end{array}$ \\
\hline II:45 am & $\begin{array}{l}\text { FACILITATED DISCUSSION } \\
\text { - Participants discuss key questions about the representative grid "architecture" and local priorities. }\end{array}$ \\
\hline $12: 30 \mathrm{pm}$ & LUNCH BREAK (ON YOUR OWN) \\
\hline $\mathrm{I}: 30 \mathrm{pm}$ & $\begin{array}{l}\text { REVIEW AND UPDATE ON IEEE } 1547 \\
\text { - } \quad \text { Ravi Subramaniam, IEEE Standards Association }\end{array}$ \\
\hline $2: 00 \mathrm{pm}$ & $\begin{array}{l}\text { OPPORTUNITIES FOR IMPROVING INTEROPERABILITY IN THE WEST } \\
\text { Panelists: } \\
\text { - } \quad \text { Adam Danise, Public Utilities Commission of Nevada } \\
\text { - } \quad \text { Sameer Kalra, PG\&E } \\
\text { - } \text { Devin Hampton, UtilityAPI } \\
\text { - } \quad \text { Anne Smart, ChargePoint } \\
\text { - } \quad \text { Lorenzo Kristov, Independent Consultant } \\
\text { - } \quad \text { Moderator: Chris Villarreal, Plugged In Strategies }\end{array}$ \\
\hline 3:00 pm & BREAK \\
\hline $3: 15 \mathrm{pm}$ & $\begin{array}{l}\text { FACILITATED DISCUSSION: CAPTURING PARTICIPANT INSIGHTS INTO GRID OPERATIONS, ECONOMICS, } \\
\text { AND INTEROPERABILITY } \\
\text { - How does interoperability relate to local operations? } \\
\text { - What is needed to improve interoperability? } \\
\text { - How does interoperability affect the customer? }\end{array}$ \\
\hline $4: 15$ pm & Conclusion AND Next Steps \\
\hline $4: 30$ pm & ADJOURN \\
\hline
\end{tabular}


Northeast Region: November 29, 2018, in Warwick, Rhode Island

\begin{tabular}{|c|c|}
\hline & $\begin{array}{l}\text { New England Regional Roundtable: } \\
\text { amework and Roadmap of Smart Grid Interoperability Standards } \\
\text { Rhode Island Public Utilities Commission } \\
89 \text { Jefferson Blvd, Warwick, RI } 02888\end{array}$ \\
\hline $9: 00 \mathrm{am}$ & $\begin{array}{l}\text { WELCOME AND AGENDA } \\
\text { - Honorable Margaret Curran, Chairperson, Rhode Island Public Utilities Commission }\end{array}$ \\
\hline 9:30 am & $\begin{array}{l}\text { KEYNOTE } \\
\text { - } \quad \text { Mark Knight, Chief Engineer, Electricity Infrastructure Group, Pacific Northwest National Laboratory }\end{array}$ \\
\hline $10: 15 \mathrm{am}$ & BREAK \\
\hline $10: 30 \mathrm{am}$ & $\begin{array}{l}\text { REgULATION IN FocUS: THE VIEW OF THE REGULATOR } \\
\text { - Honorable Katie Dykes, Chair, Connecticut Public Utilities Regulatory Authority }\end{array}$ \\
\hline $11: 00 \mathrm{am}$ & $\begin{array}{l}\text { PRIMER ON NIST SMART GRID CONCEPTUAL MODELS } \\
\text { - Avi Gopstein, NIST }\end{array}$ \\
\hline II:30 am & $\begin{array}{l}\text { FACILITATED DISCUSSION } \\
\text { - Participants discuss key questions about the representative grid "architecture" and local priorities. }\end{array}$ \\
\hline $12: 30 \mathrm{pm}$ & LUNCH BREAK (ON YOUR OWN) \\
\hline $\mathrm{I}: 30 \mathrm{pm}$ & $\begin{array}{l}\text { REVIEW AND UPDATE ON IEEE } 1547 \\
\text { - Jason Allnutt, IEEE Standards Association }\end{array}$ \\
\hline $2: 00 \mathrm{pm}$ & $\begin{array}{l}\text { PERSPECTIVES ON THE BENEFITS OF SMART GRID INTEROPERABILITY } \\
\text { Panelists: } \\
\text { - Peter Kelly-Detwiler, Northbridge Energy Partners LLC } \\
\text { - Matt Nelson, Massachusetts Department of Public Utilities } \\
\text { - Matthew McClary } \\
\text { - Jim Perkinson, National Grid } \\
\text { - } \quad \text { Henry Yoshimura, New England ISO } \\
\text { - Moderator: Chris Villarreal, Plugged In Strategies }\end{array}$ \\
\hline 3:00 pm & BREAK \\
\hline $3: 15 \mathrm{pm}$ & $\begin{array}{l}\text { FACILITATED DISCUSSION: CAPTURING PARTICIPANT INSIGHTS INTO GRID OPERATIONS AND } \\
\text { ECONOMICS } \\
\text { - How does interoperability relate to local operations? } \\
\text { - What are the constraints that limit asset utilization? } \\
\text { - How can interoperability improve return on investment? } \\
\text { - What steps can be taken to maximize device and infrastructure usefulness over its } \\
\text { physical lifetime? }\end{array}$ \\
\hline $4: 15 \mathrm{pm}$ & ConClusion AND NeXt Steps \\
\hline 4:30 pm & ADJOURN \\
\hline
\end{tabular}




\section{Appendix C: Review Update on IEEE 1547}

A session in each regional workshop provided a review and update on IEEE 1547-2018, the technical standard for the interconnection and interoperability of distributed energy resources with associated electric power systems interfaces. The standard is described on the IEEE 1547-2018 homepage as follows:

"The technical specifications for, and testing of, the interconnection and interoperability between utility electric power systems (EPSs) and distributed energy resources (DERs) are the focus of this standard. It provides requirements relevant to the performance, operation, testing, safety considerations, and maintenance of the interconnection. It also includes general requirements, response to abnormal conditions, power quality, islanding, and test specifications and requirements for design, production, installation evaluation, commissioning, and periodic tests. The stated requirements are universally needed for interconnection of DER, including synchronous machines, induction machines, or power inverters/converters and will be sufficient for most installations. The criteria and requirements are applicable to all DER technologies interconnected to EPSs at typical primary and/or secondary distribution voltages. Installation of DER on radial primary and secondary distribution systems is the main emphasis of this document, although installation of DERs on primary and secondary network distribution systems is considered. This standard is written considering that the DER is a $60 \mathrm{~Hz}$ source."

The standard started in 2013 as a 13-page technical document designed to be technologically neutral (e.g., can be used for solar wind, etc.) and devoid of policy considerations. It was first amended in 2014 and revised heavily in 2018. It is not applicable to transmission networks and is not meant to be a design handbook, and application guide, or an interconnection agreement.

The 2018 version included "interoperability" and "interfaces" in the standard title for the first time. Section 10 of the standard is focused on interoperability, with four categories of exchangeable information defined-nameplate, configuration, monitoring, and management. Cybersecurity is called out as important but is not specifically addressed in this standard. The original standard set a $10 \mathrm{MVa}$ (arbitrary) limit, but that has been removed in the more recent revision. The new version also includes functionality requirements for inverters ("shall have"), as opposed to more lenient expectations for inverters in the prior version ("may have").

The standard introduces a new term - Authority Governing Interconnection Requirements (AGIR), an entity which may be a utility or municipality that should determine the applicability of the standard. The standard takes into account both normal and abnormal conditions for DERs.

IEEE is accelerating educational programs to make sure those doing the commissioning are well-trained.

The IEEE Conformity Assessment Program (ICAP) is a program on commissioning aspects. IEEE is also trying to include UL 1741 and apply it at commissioning. IEEE 1547 is 
currently commissioning pilot studies and is still accepting applications for new pilots. There is no IEC equivalent on interconnection. IEEE 1547.1, the companion conformance testing standard for IEEE 1547, is not complete yet but is expected to be published in 2020. 


\section{Appendix D: Acronyms}

\begin{tabular}{|c|c|}
\hline ADR & Automated Demand Response \\
\hline AMI & Advanced Metering Infrastructure \\
\hline ANSI & American National Standards Institute \\
\hline C\&I & Commercial and Industrial \\
\hline CEC & California Energy Commission \\
\hline CIM & Common Information Model \\
\hline CIP & Critical Infrastructure Protection \\
\hline ComEd & Commonwealth Edison \\
\hline DER & Distributed Energy Resources \\
\hline DMS & Distribution Management System \\
\hline DSO & Distribution System Operator \\
\hline EISA & Energy Independence and Security Act of 2007 \\
\hline EMS & Energy Management System \\
\hline EV & Electric Vehicle \\
\hline GMLC & Grid Modernization Laboratory Consortia \\
\hline GW & Gigawatt(s) \\
\hline IEC & International Electrotechnical Commission \\
\hline IEEE & Institute of Electrical and Electronics Engineers \\
\hline ISO & Independent System Operator \\
\hline LED & Light-Emitting Diode \\
\hline MW & Megawatt(s) \\
\hline NARUC & National Association of Regulatory Utility Commissioners \\
\hline NERC & North American Electric Reliability Corporation \\
\hline NIST & National Institute of Standards and Technology \\
\hline NYSERDA & New York State Energy Research and Development Authority \\
\hline PG\&E & Pacific Gas \& Electric \\
\hline PJM & Pennsylvania-New Jersey-Maryland Interconnection \\
\hline PNNL & Pacific Northwest National Laboratory \\
\hline PSC & Public Service Commission \\
\hline PUC & Public Utilities Commission \\
\hline PUF & Physical Unclonable Function \\
\hline PV & Photovoltaic(s) \\
\hline $\mathrm{R} \& \mathrm{D}$ & Research and Development \\
\hline SCADA & Supervisory Control and Data Acquisition \\
\hline SDO & Standards Development Organization \\
\hline SGIP & Smart Grid Interoperability Panel \\
\hline var & Volt-Ampere Reactive \\
\hline
\end{tabular}




\section{Appendix E: Useful Links}

- NIST Smart Grid Framework homepage:

o https://www.nist.gov/engineering-laboratory/smart-grid/smart-grid-framework

- Smart Grid Interoperability Framework Workshops homepage:

o https://www.nist.gov/engineering-laboratory/smart-grid/smart-gridinteroperability-framework-workshops

- Introductory webinar:

o https://www.nist.gov/news-events/events/2018/06/nist-smart-grid-frameworkintroduction-webinar)

- Testing and Certification Landscape document:

o https://www.nist.gov/sites/default/files/documents/2018/06/25/draft_tc_landsc ape_evaluation_final.pdf

- Interoperability Profiles Description document:

o https:/www.nist.gov/document/draftinteroperabilityprofiledescriptionfinalpdf.

- "Update of the NIST Smart Grid Conceptual Model” white paper. November 8, 2018 (Third discussion DRAFT).

o https://www.nist.gov/document/draftsmartgridconceptualmodelupdatev3pdf.

- “Developing an Ontology for the Smart Grid” white paper. November 8, 2018

(DRAFT).

o https://www.nist.gov/document/draftontologyforthesmartgridv2pdf.

- “New Smart Grid Interfaces Categories Assessment” white paper. November 9, 2018 (DRAFT).

o https://www.nist.gov/document/draftinterfacecategoriesassessmentpdf.

- “Interoperability Strategic Vision: A GMLC White Paper”.

o https:/gridmod.labworks.org/sites/default/files/resources/InteropStrategicVisi onPaper2018-03-29.pdf. 


\section{Appendix F: Smart Grid Communications Scenarios}

These diagrams, presented during the regional workshops, were created to complement the Conceptual Models, illustrating communications networks in smart grid scenarios based on the Conceptual Model. They will be updated based on feedback obtained during the workshops before publication in the next Framework.

\section{Legacy Utility Communication Pathways Scenario}

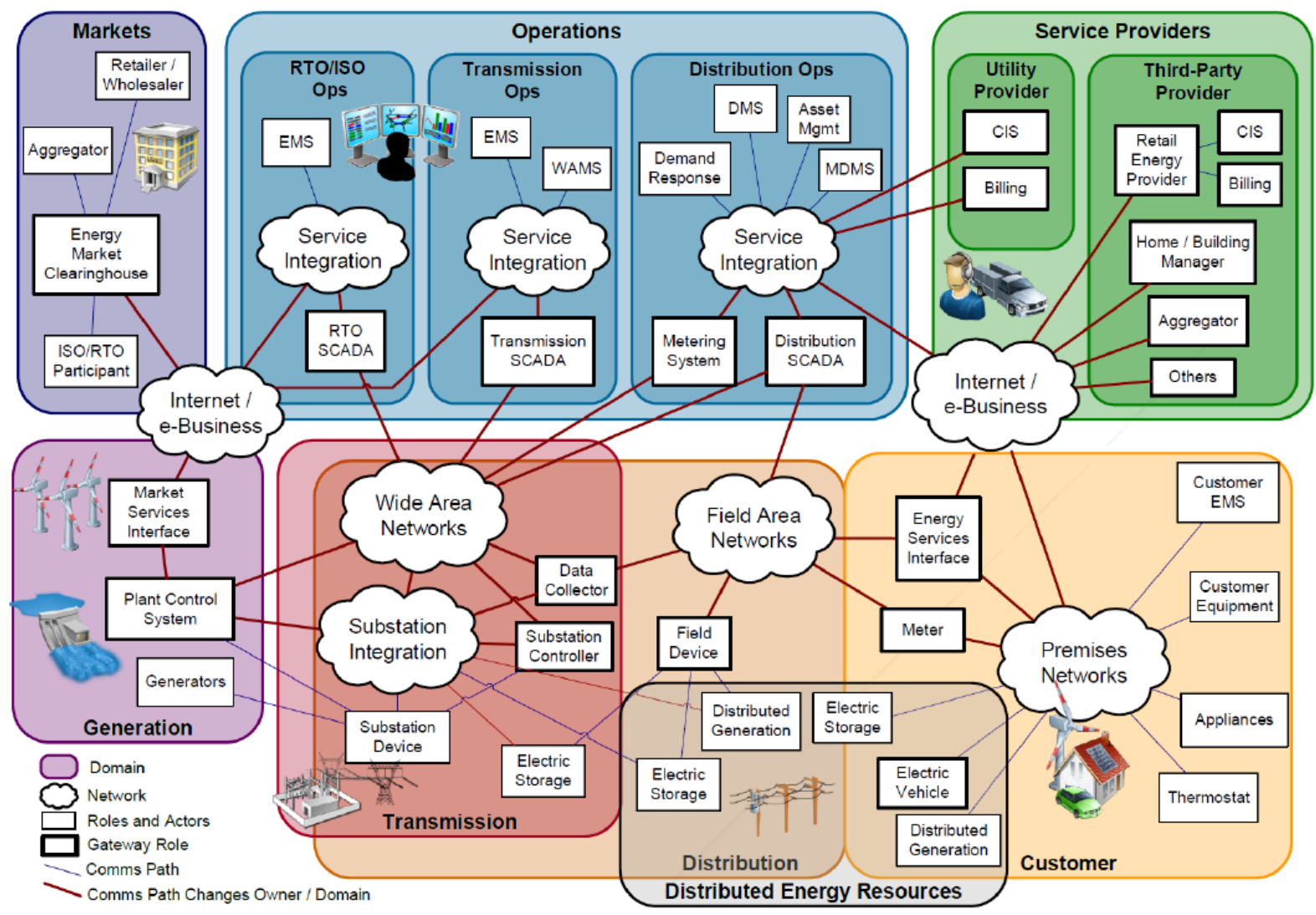




\section{High-DER Communication Pathways Scenario}

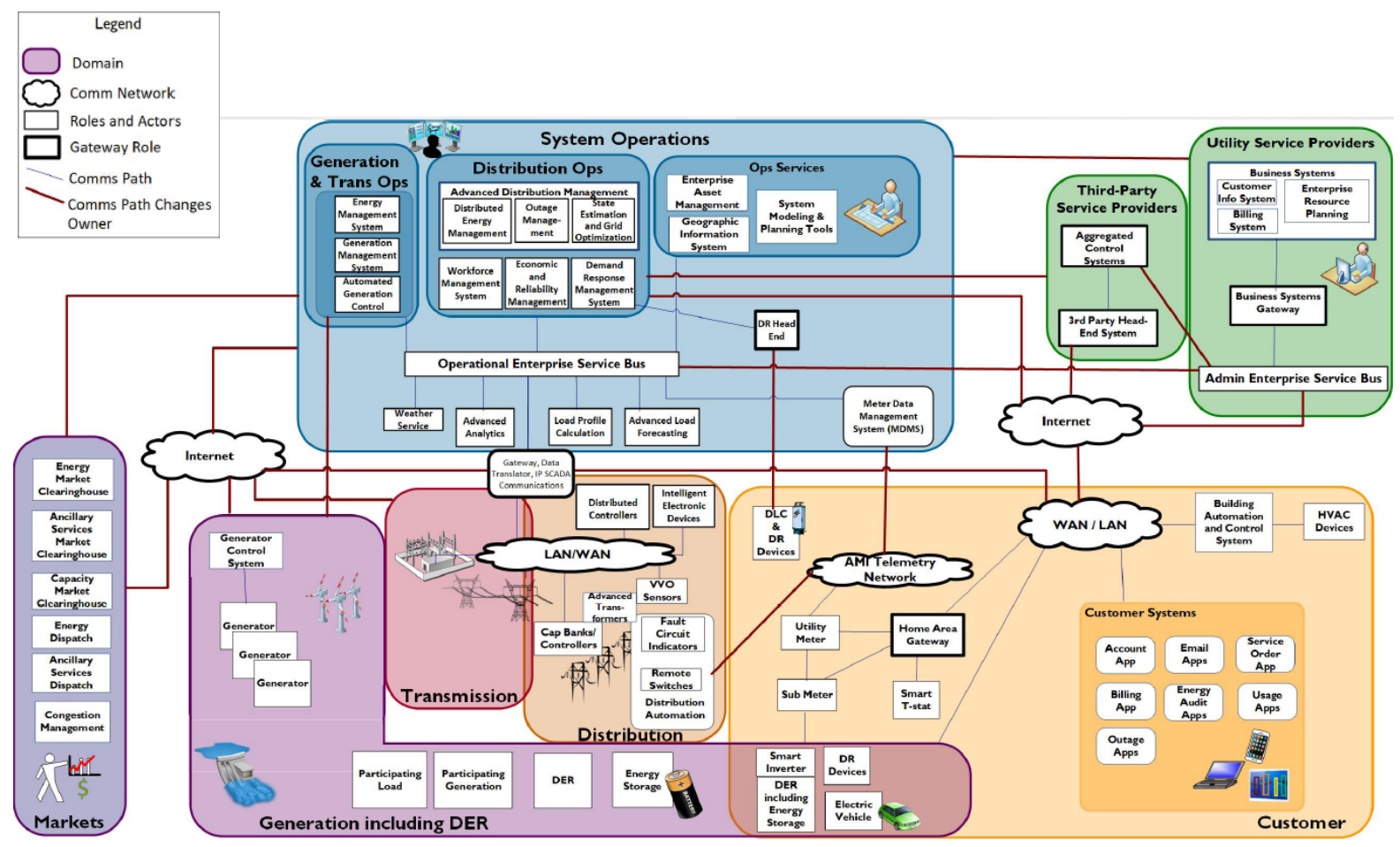

\section{Microgrid Communication Pathways Scenario}

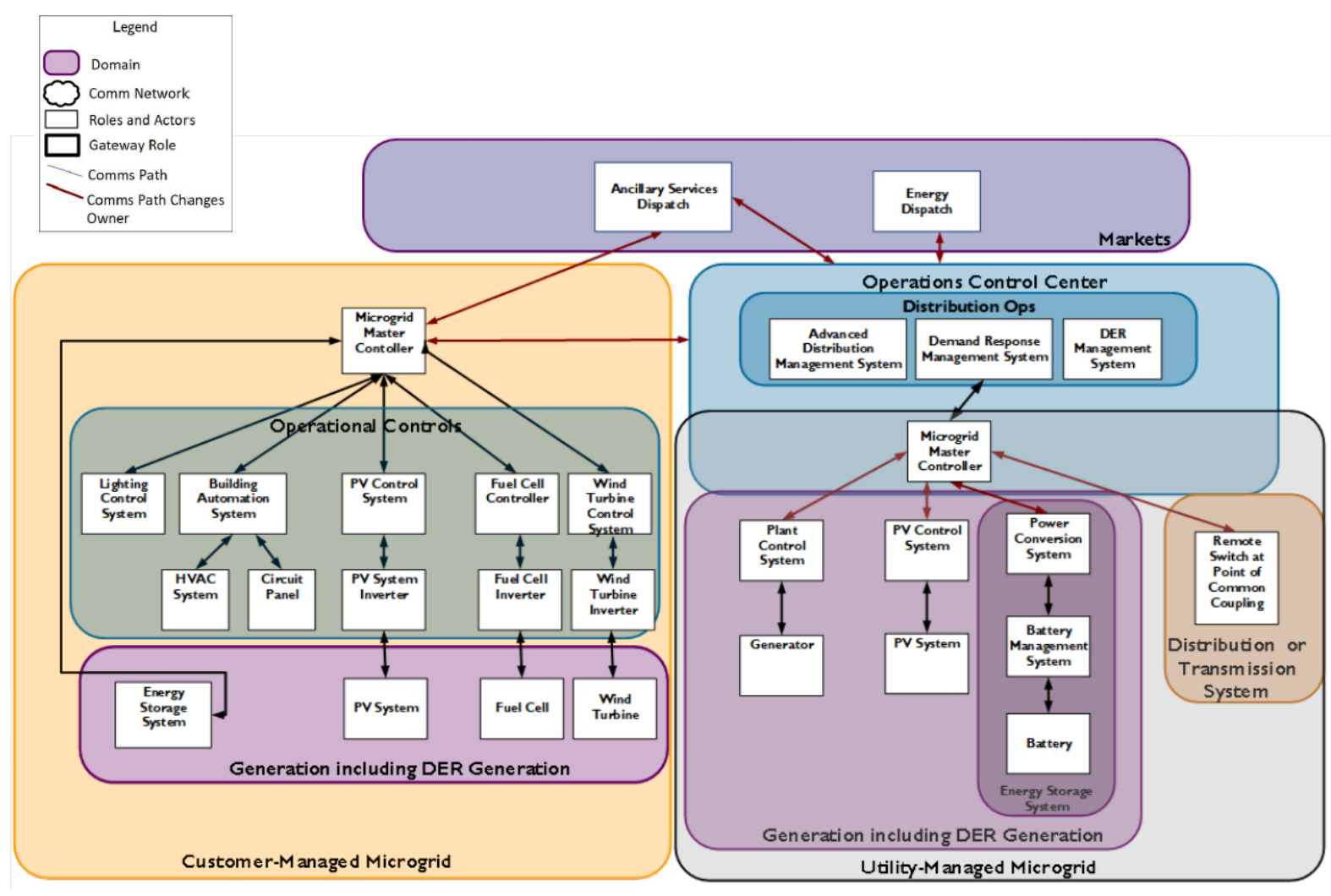




\section{Hybrid Bulk-Grid Communication Pathways Scenario}

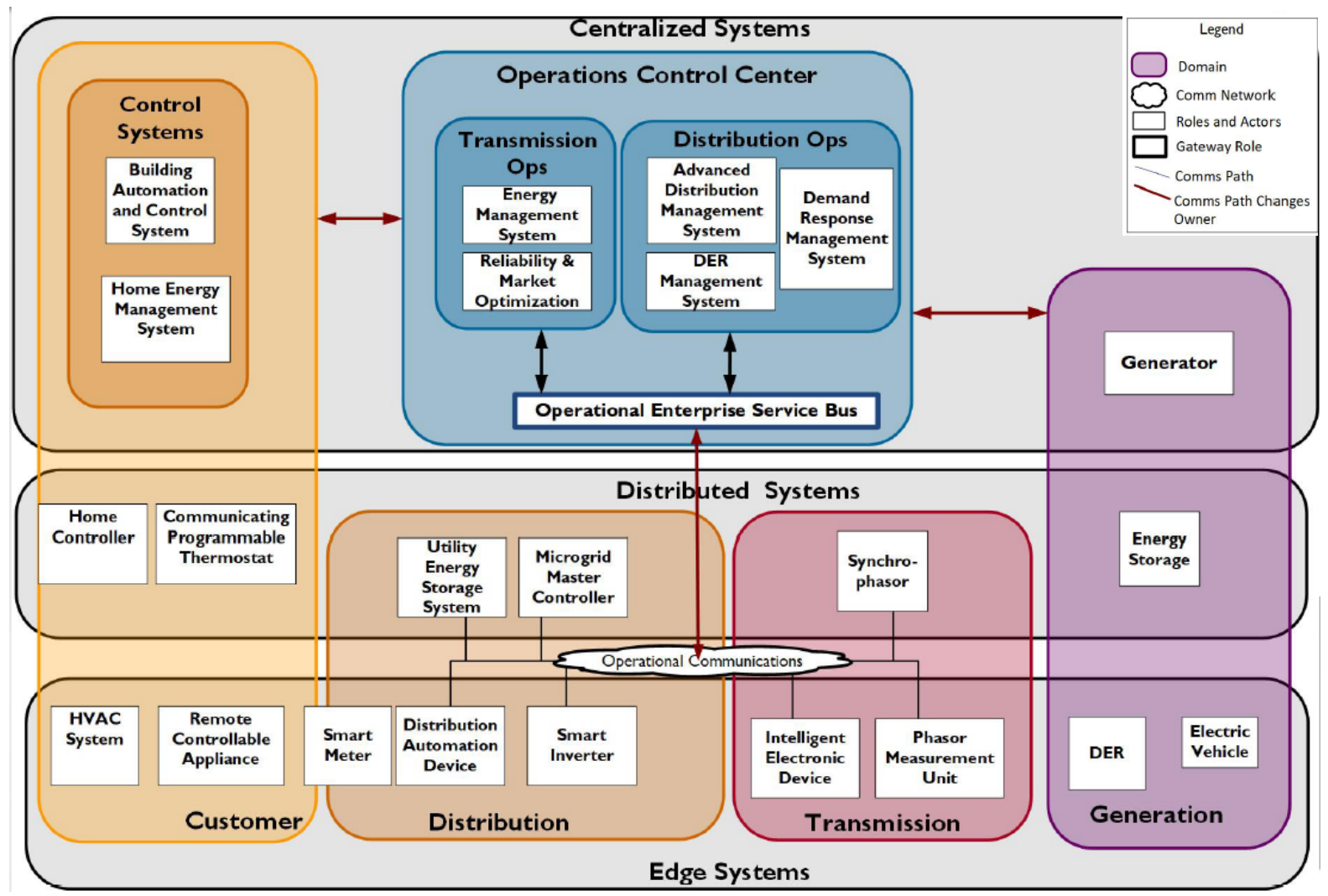

